\title{
Attention biases preferential choice by enhancing an option's value
}

\author{
Timothy J. Pleskac \\ University of Kansas \\ Shuli $\mathrm{Yu}$ \\ Judge.me \\ Sergej Grunevski \\ University of Kansas \\ Taosheng Liu \\ Michigan State University
}

\begin{abstract}
Does attending to an option lead to liking it? Though attention-induced valuation is often hypothesized, evidence for this causal link has remained elusive. We test this hypothesis across two studies by manipulating attention during a preferential decision and its perceptual analog. In a free-viewing task, attention impacted choice and eye movement pattern in the preferential decision more than during the perceptual analog. Similarly, in a controlledviewing task, attention had a larger effect on choice in the preferential decision than its perceptual analog. Across these experimental manipulations of attention, choice and eye-tracking data provide converging evidence that attention enhances value, and computational modeling further supports this attention-induced valuation hypothesis. A possible explanation for our results is a normalization mechanism where attention induces a gain modulation on an option's representation at both the sensory and value processing levels.
\end{abstract}

Keywords: attention, value, decision, preference, perception

LADY CAPULET. Speak briefly, can you like of Paris' love?

De-identified data and analyses are at the Open Science Framework: OSF Anonymous Peer Review Link. This research was supported in part by a grant from the National Institutes of Health (R03DA033455) and a grant from the National Science Foundation (1756092) to T.L. and T.J.P.: Conceptualization: T.L., S.Y., and T.J.P.; Investigation: S.Y.; Formal analysis: S.G., T.L., S.Y., and T.J.P; Writing: S.G., T.L., S.Y., and T.J.P. The data from this paper comes from Shuli Yu's dissertation. Correspondence concerning this article should be addressed to either Timothy J. Pleskac (e-mail: pleskac@ku.edu) or Taosheng Liu (e-mail: tsliu@msu.edu). Version 7.0, 7/21/2022. 
JULIET. I'll look to like, if looking liking move but no more deep will I endart mine eye than your consent gives strength to make it fly.

- Romeo and Juliet, Shakespeare, 1853, 1.3.100-103

In Romeo and Juliet (Shakespeare, 1853), Lady Capulet encourages Juliet to gaze at her potential suitor's face, take delight in his beauty, and in so doing, hopefully, grow to like him and accept his love. Juliet, however, is hesitant. Ever cautious, she agrees to take a chance and look at her suitor, Paris. But in the same breath, she exercises restraint perhaps acknowledging that looking too deeply could render her vulnerable to a torrent of unchecked emotions. Can looking lead to liking as Lady Capulet hopes and Juliet worries? Certainly, they are not the first people to consider the possibility that attending to an option might help garner value for it, nor are they the last. Would-be suitors, marketers, politicians, and many others have acted on this hypothesis.

There are several indicators of a potential link between attention and value. It would seem to be at the heart of the mere-exposure effect where repeated exposure to an option appears to enhance attitudes toward it (Zajonc, 1968, 2001). It also seems consistent with the gaze-cascade effect where in the final moments before a preferential decision, people grow more likely to look at the item they are about to choose (Shimojo et al., 2003; Simion \& Shimojo, 2006, 2007). More recently, studies using computational models of decision making have explicitly proposed a mechanism where visual attention enhances option valuation, which we refer to as the attention-induced valuation (AIV) hypothesis. Using eye fixation as a proxy for attention, these models assume that attention magnifies the subjective value of the attended-to-option (Krajbich et al., 2010; Krajbich \& Rangel, 2011; Thomas et al., 2019), and can account for the relationship between fixation and choice such as the gaze cascade effect and faster response times in higher vs. lower-valued options (Smith \& Krajbich, 2019).

To further appreciate the AIV hypothesis, we first need to establish a more precise definition of attention. Visual attention refers to the cognitive and neural mechanisms that allow organisms to select environmental information for prioritized processing (Posner, 1980; Desimone \& Duncan, 1995; Carrasco, 2011). Such selection is necessitated by peripheral factors such as the highly non-uniform acuity across the visual field and central factors such as limits in working memory and executive control (Curcio et al., 1990; Marois \& Ivanoff, 2005). During natural viewing, the eyes move 3-4 times per second to sample information from a scene, and the fixation pattern is often used to infer where attention is allocated (Henderson \& Hollingworth, 1998; Kowler, 2011). In addition to voluntarily selecting information based on task goals, salient objects and events can involuntarily draw attention (Yantis, 1993; Theeuwes, 2004). In the context of value-based decision making, several studies have found that salient stimuli were more likely to be chosen in preferential decisions, which is also consistent with the AIV hypothesis (Tsetsos et al., 2012; Zeigenfuse et al., 2014; Towal et al., 2013). Still, other studies have shown that changes in gaze duration (Armel et al., 2008; Shimojo et al., 2003), or prompting decisions when people are looking at certain options (Pärnamets et al., 2015; Ghaffari \& Fiedler, 2018; Tavares et al., 2017; H.-Z. Liu et al., 2020) can all impact choice, in a manner consistent with the AIV hypothesis.

However, two important limitations with these results stymie the conclusion that attention induces value. The first is that eye fixation and attention are not the same (see 
also Mormann \& Russo, 2021). Attention is an internal state of the cognitive and neural system, whereas eye movement is a motor output of the system. As such, using the motor act of looking to infer the cognitive state of attention can be problematic. To illustrate, consider the scenario where people choose by reaching for the item with their hand. We will certainly recreate a "hand cascade" effect such that people are more likely to reach for the option they ultimately choose in the final moments before a decision. Yet, it would be untenable to maintain that the arm reach is an indication of attention and induces a preference; instead, most would agree that reaching is a consequence of intention and preference. The same problem occurs for using looking or gaze to measure attention. Indeed, although attention and gaze are generally correlated, it is also well known that they do not always co-localize. For example, people can certainly attend to a different location than the currently fixated location (Von Helmholtz, 1925; Posner, 1980; Carrasco, 2011), and sometimes people even fail to process visual information that is present in the currently fixated location (Mack \& Rock, 1998; Simons \& Chabris, 1999).

Even if eye fixation can be used as a proxy of attention, a second limitation arises: attention also impacts perception and subjective appearance. Generally speaking, attention increases the perceived salience of the attended object. For example, attending to an object makes it appear higher in contrast (Carrasco et al., 2004; T. Liu et al., 2009), more saturated in color (Fuller \& Carrasco, 2006), moving faster (Turatto et al., 2007), and alters other feature appearance (Carrasco \& Barbot, 2019). Because perception provides stimulus information to all higher-level cognitive processes including valuation, an effect on an earlier stage (perception) can propagate to later stages (valuation). Thus, most of the results supporting the AIV hypothesis can be potentially attributed to an effect of attention on perception instead of value representation. For instance, suppose attention can be likened to a filter that filters out the unattended object (Broadbent, 1958; Everling et al., 2002), then it would be no surprise if attention also impacts valuation because it essentially removes the unattended object such that valuation can only be based on the attended object. Such a scenario is still informative for our understanding of value-based decision making. However, we would argue that under this scenario, attention does not induce valuation; instead, an apparent effect of attention on valuation may be due to its impact on the perceptual system.

Here, we aim to overcome these challenges to better test the AIV hypothesis. First, we manipulated attention instead of only measuring gaze behavior. We did so in two ways. In study 1 , we used a free viewing paradigm where participants could freely look at different options and we presented a brief peripheral cue to attract attention involuntarily. In study 2, we used a controlled viewing paradigm where we showed options one at a time and manipulated the duration in which the options were shown. Second, we sought to disentangle any effect of attention on valuation from its effect on perception by comparing preferential decisions with a matched perceptual decision baseline. Specifically, we used the Flash Gambling Task (FGT; Zeigenfuse et al., 2014; Pleskac et al., 2019) and its perceptual analog, which offers precise control of the option values. In this task, participants were asked to choose between two options, each consisting of an array of dots. In one or both options, the number of dots was determined by a draw from a normal distribution and was dynamically updated at a rapid rate. In the preferential frame, participants were told that each dot represented a fixed reward and they should choose the option with a higher instantaneous reward; in the perceptual frame, participants were told to choose the option 
that has a higher number of dots on average (see also Dutilh \& Rieskamp, 2016). These task settings were employed to align with the typical conceptual definitions of perceptual and preferential decisions where a perceptual decision typically has an objectively correct answer (Hanks \& Summerfield, 2017), but a preferential decision does not have an objectively correct answer (Luce \& Raiffa, 1957; Pleskac et al., 2015). Accordingly, the feedback and payoff for these two conditions also reflected these framings. In the preferential frame, the feedback and payoff were based on a single sample from the chosen option modeling a risky gamble. In the perceptual frame, the feedback and payoff were based on the mean of the underlying distribution, which was objectively defined. This design allowed us to compare two decision frames with identical stimulus input, thus providing a means to isolate the effect of attention on preference over and above its effect on perception.

In addition to these two main strategies, we adopted several additional strategies to fully characterize attention's effect on preference. First, we tracked eye position in a free viewing task (Study 1), which allowed us to assess how the manipulation of attention via the peripheral cue affected the subsequent gaze pattern and the relationship between gaze and choice. Second, we used a controlled viewing task (Study 2) to assess whether eye movement per se is necessary for an impact on choice. Third, we employed computational models to help isolate how attention and gaze impact the decision process during preferential choice. To foreshadow our results, we found converging evidence supporting the AIV hypothesis that attention actively contributes to preferential decisions by enhancing an option's value.

\section{Study 1: Cued free viewing decisions}

Our first study examined preferential and perceptual decision making when participants could freely look at the different options and choose when they were ready (i.e., optional stopping). Half the participants were randomly assigned to the FGT and the other half to its perceptual analog. Across both conditions, we manipulated spatial attention towards one of the options by presenting a peripheral cue in its location or a central cue as a neutral condition. We note that the cue did not instruct participants to shift their attention or gaze, as it was non-informative regarding the correct option, and participants were made aware of this fact. Nevertheless, research in visual attention has shown that with suitable spatiotemporal characteristics, such cues attract participants' attention and eye gaze involuntarily (Theeuwes et al., 1999; Yantis, 1993). Thus, this method provided an unobtrusive way to manipulate attention. Accordingly, the AIV hypothesis predicts that during a preferential choice, participants would be more likely to choose the cued option at the expense of maximizing expected value due to having a higher gaze bias. Conversely, during a perceptual choice, participants would be less likely to exhibit a gaze bias and consequently more likely to choose the option with the higher expected value. We did not preregister this study.

\section{Method}

Participants. A total of 61 participants (31 for the preference and 30 for the perceptual condition) were recruited from the Michigan State University community. They were paid $\$ 12$ and a $\$ 1-5$ performance bonus to take part in a single 1.5 hour session of the study. Michigan State University's Institutional Review Board approved the study. 
Design. The study used a 2 (decision frame) $\times 3$ (cue) $\times 5$ (mean difference) mixed design. The frame (preference vs perceptual) varied between subjects; the attention manipulation (left vs central vs right cue) and mean difference ( 5 levels) both varied within subjects across trials. The mean difference (hereafter referred to as relative value) corresponds to the difference in the mean number of dots between options (right minus left option: -40, -20, 0, 20,40), made up from six combinations of option pairs (see below).

Flash Stimulus. The stimuli were generated in MATLAB using Psychophysics Toolbox Version 3 (Brainard, 1997). Participants viewed two circular display options on an LCD monitor refreshed at $60 \mathrm{~Hz}$ (Figure 1). Each display contained two circular fields of dynamically updating white dots on a black background with a diameter of $6.1^{\circ}$ visual angle, with one located $6.75^{\circ}$ to the left of a red central fixation and the other $6.75^{\circ}$ to the right.

The dot display changed every $50 \mathrm{~ms}(20 \mathrm{~Hz})$. At each update, a new sample of dots was drawn from an underlying distribution and positioned at randomly generated locations within the circular field. There were four different display options to manipulate the number of dots in each sample: Three options were a Gaussian distribution, truncated at the lower bound of 0 , with a mean of either 110, 130 or 150 dots, and a standard deviation of 40 dots. The fourth option always had a fixed number of 130 dots, with the locations of all dots randomly updated every $50 \mathrm{~ms}^{1}{ }^{1}$ These four options were factorially combined to yield six unique pairs of options, resulting in 5 levels of relative value difference $(-40,-20,0,20,40$ dots) and 3 levels of unsigned relative value difference (0,20 or 40 dots) between options. The option's location (left/right) was randomly assigned on each trial.

Procedure. Participants were randomly assigned to either the preference or perceptual frame. In order to help participants understand the task, they were told to imagine that the two options were two ponds with fish swimming in them and the dynamic dots represented the fish surfacing and submerging. In both tasks, participants were instructed to observe the two options to form an impression of the number of fish surfacing. In the preferential frame, they were told to choose a pond to fish from, such that they would catch the number of fish that surfaced on the next instant and be rewarded for the number of fish they caught. In the perceptual frame, they were told to choose the option that had the higher average number of fish surfacing in each trial (for full instructions see OSF). Participants completed eight blocks of 90 trials (a total of 720 trials) of the FGT or its perceptual analog. During the task, participants' right eye positions were recorded with an Eyelink 1000 system (SR Research, Ontario, Canada) at $500 \mathrm{~Hz}$.

In each trial, participants started by viewing a fixation dot for $500 \mathrm{~ms}$ in the center of the screen (Figure 1). Then a cue in the form of a red dot of $0.75^{\circ}$ appeared either in the center (neutral cue: on $1 / 3$ of the trials) or periphery ( $9^{\circ}$ to the left on $1 / 3$ of trials; $9^{\circ}$ to the right on $1 / 3$ of trials) of the screen for $67 \mathrm{~ms}$ ( 4 frames). The location of the cue was randomly determined on each trial and had the purpose of either keeping participants'

\footnotetext{
${ }^{1}$ We included the $S D=0$ (certain) option as an exploratory manipulation. Our past studies (Pleskac et al., 2019; Zeigenfuse et al., 2014) with the FGT had only paired certain and uncertain options, which could introduce some perceptual asymmetries. Here, the only difference we found was that participants were more sensitive to relative value differences when an uncertain option was paired with a certain option than it was paired with another uncertain option. This sensitivity was a bit higher in the perceptual condition. There was no credible interactions between the certainty/uncertainty option and the attentional cue and thus we collapsed across this exploratory manipulation in all analyses reported here.
} 


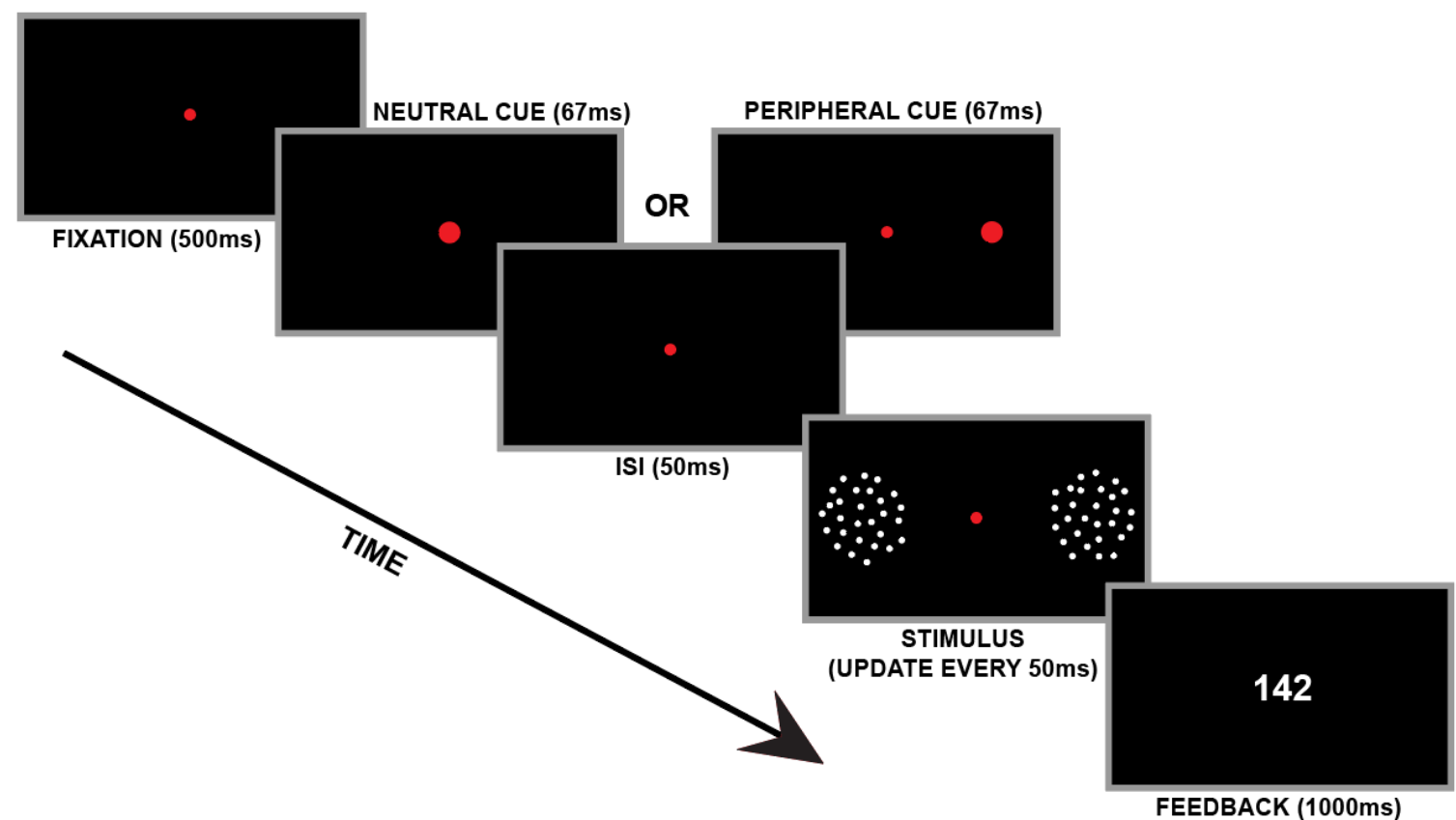

Figure 1. Trial schematic for Study 1. Each trial contained a central fixation point, a brief cue, an inter-stimulus interval (ISI), and two dot stimuli. Participants were free to look at the stimulus until they chose an option via a key press, in either a preferential or perceptual decision frame. They then received appropriate feedback message for each decision frame.

attention to the center (neutral cue) or orienting their attention toward a particular option (peripheral cue). Participants were informed that the cue did not indicate the correct option and they should ignore it entirely. After a second fixation in the center for $50 \mathrm{~ms}$ (3 frames), the two flash stimuli appeared on the left and right. The stimuli remained on the screen until participants indicated their choice by pressing a key (left option: "1" on the number pad; right option: "2" on the number pad) with their right hand.

After recording a choice, participants received feedback about their choice. In the preferential frame, they were told the number of dots (fish) that would have appeared in the next frame. In the perceptual frame, they were told the average number of dots in the chosen option. Both conditions were incentivized. In the preferential frame, the total number of dots that were sampled (i.e., fish caught) were accumulated across the trials as a score while in the perceptual frame the average number of dots from the chosen option were accumulated as a score. The score was exchanged for a bonus payout for $\$ 1-5$ at the end of the session, with participants in the perceptual frame $(M=\$ 3.94$, $S D=0.07)$ earning slightly more than those in the preferential $(M=\$ 3.90, S D=0.09)$ frame $\left(M_{D}=0.04[0.01,0.08]\right)$.

Data Analysis. Our statistical analyses employed a multi-level modeling approach using Bayesian estimation techniques (Gelman et al., 2013; Kruschke, 2014). In each of the analyses, Markov Chain Monte Carlo (MCMC) methods were used to generate estimates from the posterior distribution of each parameter. All chains were inspected for the representativeness of the posterior distribution both visually and with the Gelman-Rubin statistic. We also inspected the autocorrelation within chains to confirm their ability to 
provide stable and accurate estimates of the distributions. In general, for the reported parameter values we sought to have an effective sample size of approximately 10,000. In reporting results from the models, we report the mean of the posterior distribution of the parameter or statistic of interest and the $95 \%$ Highest Density Interval (HDI).

Pre-processing of behavioral and eye-tracking data. The raw eye-tracking data (horizontal and vertical positions over time, $x(t)$ and $y(t)$ ) was segmented by each trial and resampled every $25 \mathrm{~ms}(40 \mathrm{~Hz})$ to produce two sets of eye movement trajectories: one was time-locked to stimulus onset and the other to the time of response. We first classified each sample of eye position into three zones using the horizontal position $(x)$, because the stimuli were horizontally displaced. The zones were defined in terms of degrees from the center of the screen: Left $\left(x<-2^{\circ}\right)$, Center $\left(-2^{\circ} \leq x \leq 2^{\circ}\right)$, and Right $\left(x>2^{\circ}\right)$. This three-zone partition was used for the gaze dwell time regression analysis. ${ }^{2}$

For the drift-diffusion model analysis, the Left and Right zone designations were preserved; however, if a gaze sample was located in the Center zone, it was recorded as being equally split between the Left and Right zones. Relative gaze dwell time was calculated as the amount of time the gaze was in each zone divided by the respective trial response time. Normalized dwell times were derived for the Left and Right zones by dividing the relative dwell time for each zone by the sum of the relative dwell time in the Left or Right, thereby excluding the Center.

For the gaze cascade regression analysis, we denoted a binary status variable that tracked whether a participant's gaze was focused on the eventually chosen option. As the gaze cascade refers to the likelihood of fixating on the eventually chosen option time-locked to the choice, the regression analysis focused on the final $250 \mathrm{~ms}$ of gaze samples, up to the point of the choice.

We removed trials where participants selected an incorrect button (chose neither left nor right option). Consistent with our previous work with the FGT (Pleskac et al., 2019), we also removed trials with responses faster than $0.25 \mathrm{~s}$. Responses faster than this timing threshold showed no sensitivity to the relative value and thus we classified them as guessing. The average number of trials that were removed under both of these cutoffs was $15.2(2.12$ $\%$ total) $(\mathrm{SD}=44.2)$ per participant. To minimize the chances that we included trials where participants were distracted, we also removed trials with response times longer than 5 seconds from all analyses. The average number of trials that exceeded the $5 \mathrm{~s}$ cutoff was $13.8(1.96 \%$ total) $(\mathrm{SD}=27.3)$ per participant. For analyses involving gaze data, trials were removed if more than $50 \%$ of the eye-tracking samples were missing, which excluded an average of 43.0 trials per participant $(6.23 \%$ total) $(\mathrm{SD}=70.75)$.

Psychometric function. Choice behavior was modeled with a hierarchical fourparameter logistic psychometric function (Macmillan \& Creelman, 2004; Wichmann \& Hill, 2001). Accordingly, the probability of choosing the right option is a function of the relative value, $d$,

\footnotetext{
${ }^{2}$ Setting three areas of interest departs from other studies of decision making that used just two areas centered around each option (e.g., Shimojo et al., 2003). We took this approach after noting that a significant proportion of fixations $(\mathrm{M}=39.1 \%$; $\mathrm{SD}=12.0 \%$, range of $8.72-75.3 \%$ across participants $)$ did not fall directly on the two stimuli, especially at the beginning of the trial. Instead, they fell in the center region. Hence, we took a more conservative approach by using three broad categories: left, right, and center. On average, participants looked left $34.1 \%$ of the time, center $30.2 \%$ of the time, right $34.0 \%$ of the time, and off the screen $1.7 \%$ of the time.
} 


$$
\operatorname{Pr}(\text { ChooseRight })=\gamma+(1-\gamma-\lambda) \frac{1}{1+\exp \frac{-(d-\mu)}{\theta}} .
$$

The $\gamma$ and $\lambda$ parameters determine the lower and upper asymptote of the psychometric function and account for the base rate of choosing right or left options, respectively. ${ }^{3}$ The asymptote parameters $\gamma$ and $\lambda$ were free to vary between decision frames, but fixed across the cue conditions for a given decision frame. The parameter $\mu$ is the threshold parameter $(\mu)$ and determines the location where the point of subjective equality (the halfway point between the lower and upper asymptotes of the psychometric function) is in terms of the relative value $d$. We used the threshold parameter $\mu$ to measure the effect of the attentional manipulation on choice behavior. The parameter $(\theta)$ is a slope parameter that determines how the probability of choosing the right option changes with the relative value. We allowed both parameters to vary between the decision frame and cue conditions. To estimate the sensitivity, or the ability to discriminate one option from another in terms of the relative value, we calculated the slope of the psychometric function at the threshold.

Regression models. We used a multilevel model to examine the effect of the experimental manipulations on gaze variability (standard deviations of trial-level horizontal gaze position), gaze dwell time (normalized, trial-level gaze proportions to the Right-logit transformed), gaze cascade (binary gaze status of looking at the eventually chosen option for each sample in a $250 \mathrm{~ms}$ interval before choice), and response times (inversed and standardized). Within-subjects variables were the relative value (mean dot difference divided by 20 to yield $-2,-1,0,1,2$ ), unsigned relative value (absolute mean dot difference divided by 20 to yield 0,1 , and 2), and initial cue location (dummy coded left vs. center vs. right), whereas the between-subject variable, was the decision frame (dummy coded Perceptual vs. Preferential). We used a logistic link for gaze cascade data and a normal link for gaze variability, gaze dwell time, and response time data. The models were estimated using RStanArm with the standard priors (Goodrich et al., 2020), which involved generating 10 chains of 12,000 steps (2,000 steps discarded) estimated from the posterior distribution of each parameter. The predictor variables were unstandardized in regressions for all studies, and we report $b$, the unstandardized coefficient which quantifies the effect of the experimental conditions on the measured criterion values.

\section{Results}

Choice behavior. The group-level psychometric functions for each condition are shown in Figure 2 (for group level parameter estimates see Table S1). For both decision frames, as the relative value (right-left) increased, participants tended to choose the right option more often.

Threshold. Furthermore, psychometric functions for different cue conditions exhibited a systematic lateral shift. Relative to the center cue and across relative value differences, the left cue shifted the function rightward, indicating a greater difference in relative value was needed for participants to choose the right option when the left option was cued. Similarly, the right cue shifted the function leftward, indicating a smaller difference in relative

\footnotetext{
${ }^{3}$ The two asymptote parameters were necessary to account for differences at the individual participant level and to facilitate comparisons in threshold and sensitivity.
} 


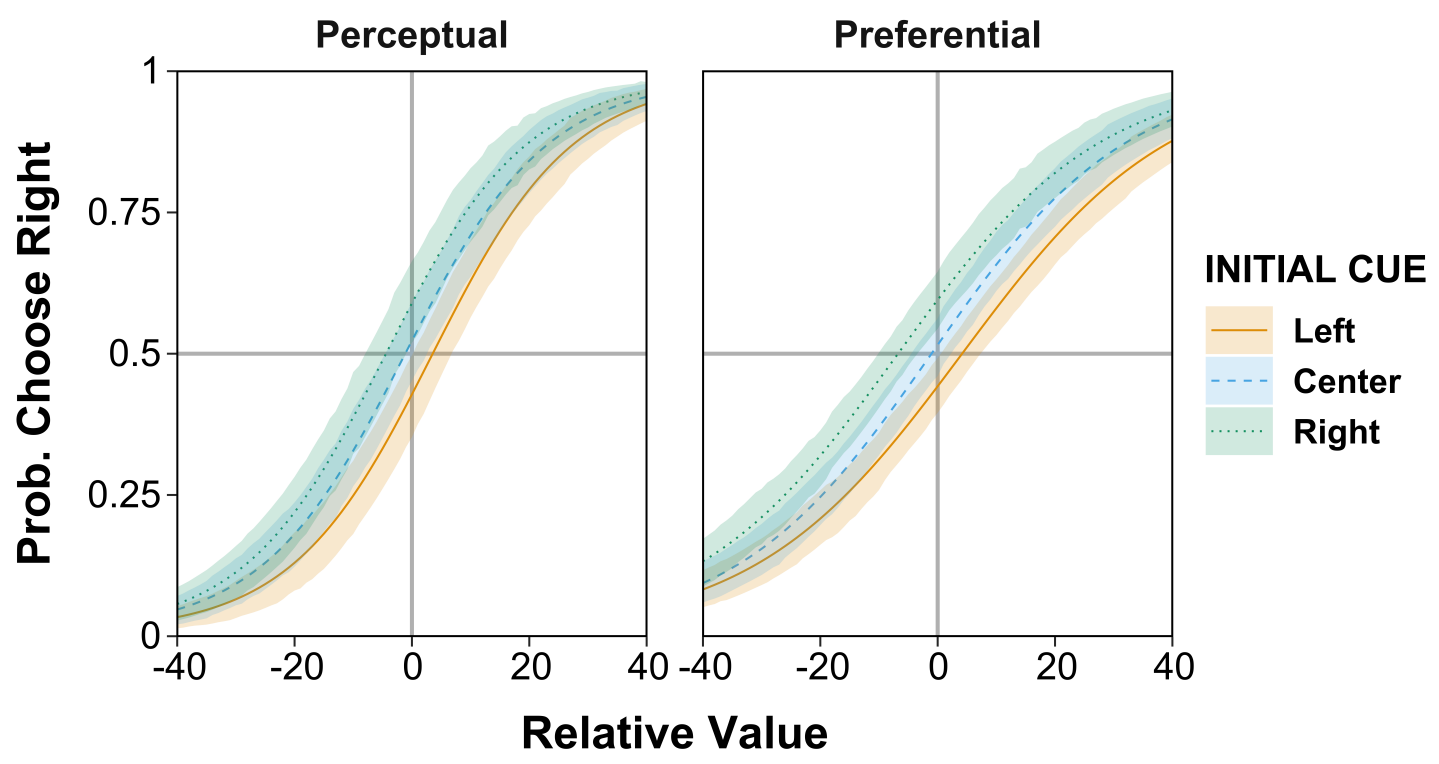

Figure 2. The probability of choosing the right option is plotted against the relative value (difference in mean dots between options, right-left). The data was conditioned by the decision frame (panels) and cue location (color). The dark lines represent the posterior predicted choice proportions with the error regions indicating their 95\% HDIs of the grouplevel posterior distributions.

value was needed for participants to choose the right option when it was cued. We can quantify the total amount of shift via the psychometric function's threshold, $\mu$. Doing so revealed a credible shift in threshold for the preferential $(M=11.00 .92[9.68,12.25])$ and perceptual frames $(M=7.98[6.82,9.07])$. Moreover, consistent with the AIV hypothesis, the cueing effect in the threshold was credibly larger in the preferential frame than in the perceptual frame $(M=3.02[1.29,4.73])$. In other words, the effect of the attentional manipulation in the preferential frame was above and beyond its effect on the perceptual frame.

Sensitivity. Figure 2 also shows that the group-level psychometric functions in the perceptual frame had a steeper slope than that in the preferential frame, suggesting a greater sensitivity to relative value in the perceptual than the preferential frame. Indeed sensitivity in the preferential frame $(M=0.0144[0.0119,0.0170])$ was lower than in the perceptual frame $(M=0.0205[0.0167,0.0248])$. This difference in sensitivity was credible $(M=-0.0061[-0.0113,-0.0016])$. As we show in the supplementary material, this difference in sensitivity was largely driven by a few individuals with extremely low sensitivity (see Supplementary Material 1.3.1).

Nevertheless, the potential difference in sensitivity between decision frames does raise an alternative explanation for the credible cueing effect: perhaps there was more uncertainty in the preferential frame (thus lower sensitivity), making participants more susceptible to the cue. We examined this further by matching the two conditions in terms of sensitivity (the details of this analysis are reported in Supplementary Material 1.3.2). Briefly, we used 


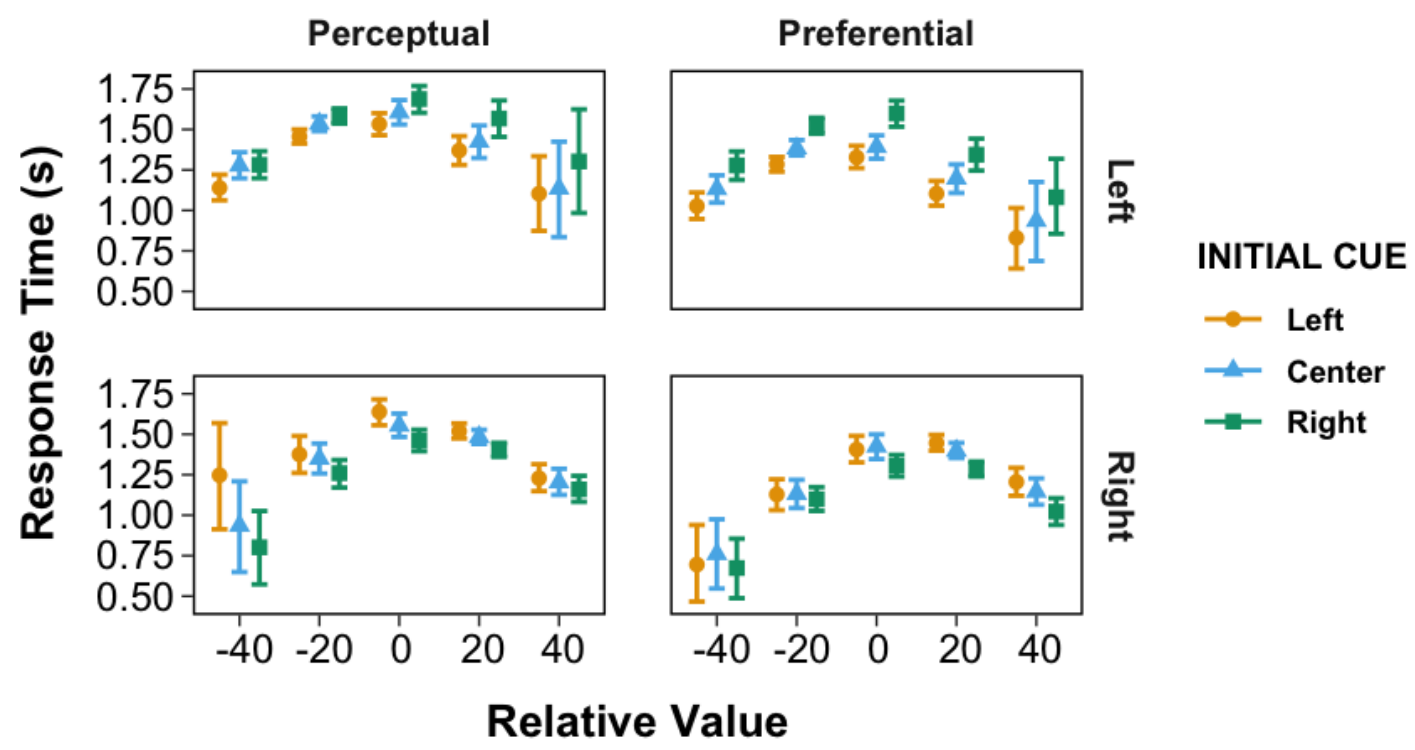

Figure 3. The posterior predicted response times are plotted against the relative value. The dots represent the posterior means of the response times, and the error bars are the $95 \%$ credible intervals of the group-level posterior distributions. The data were conditioned by the decision frame, eventual choice (panels), and cue location (color and shape). Note for the top row (left choice), negative relative values indicate correct responses for the perceptual frame and expected value maximizing response for the preferential frame. The positive relative values indicate incorrect responses for the perceptual frame and not expected value maximizing responses for the preferential frame. For the bottom row (right choice), this is reversed

the slope of the neutral condition to index the sensitivity of each participant and designed an algorithm to sub-sample the participants to obtain 20 pairs of participants from the two decision frames with similar or matching sensitivity (and thresholds) in the neutral condition. Applying the same analysis as above again revealed a credible shift in the threshold for the preferential $(M=10.21[8.88,11.52])$ and perceptual frames $(M=8.17[6.8,9.37])$. Moreover, consistent with the AIV hypothesis, the cueing effect in the threshold was credibly larger in the preferential frame than in the perceptual frame $(M=2.05[0.26,3.84])$. Crucially, because the conditions were matched in terms of sensitivity, there was no credible difference in sensitivity between decision frames $(M=-0.0026[-0.0078,0.0026])$. Together this result speaks against the idea that the cueing effect was due to differences in uncertainty between the conditions and instead helps further support the AIV hypothesis.

Response times. Group-level response times (RT) for each condition are shown in Figure 3. To assess the effect of our experimental manipulations on RT, we regressed the standardized, inverse response times against the relative value, decision frame (Perceptual vs. Preferential), cue location (Left, Center, Right), and choice (Left vs. Right), including a term for squared relative value to account for the inverted U-shape of Figure 3. Response times decreased as the relative value for an option increased (positive quadratic relative 
value term because of inverse Gaussian RTs; $b=18[16.27,19.72])$, indicating better discrimination between options as the relative values became more extreme. Response were also on average faster in the preferential $(M=1.43 \mathrm{~s}[1.21,1.65])$ than perceptual frame $(M=1.61 s[1.39,1.84] ; b=0.37[0.02,0.73])$. Again like the difference in sensitivity, when we examined the individual level response times, we found that a few individuals largely drove this difference in response time in the preference frame-by and large, the same individuals with extremely low sensitivity - responded very quickly (see Supplementary Material 1.3.1).

Just as with the difference in sensitivity, the difference in response times could suggest some differences in information processing that might explain the greater effect of cue in the preferential frame on the thresholds. Thus, we extended our matching analysis to match the perceptual and preferential groups in terms of sensitivity, threshold, and mean response times in the neutral condition. We again obtained essentially the same results in these sub-sampled participants - a larger cueing effect in the preferential than the perceptual condition (for details see Supplementary Material 1.3.2).

In addition to these effects of relative value and decision frame, the cue credibly affected response times. But, this effect depended on whether participants chose left or right (see Figure 3). The fastest responses occurred when cue and choice were congruent: when the left option was cued, the left choice was faster $(M=1.46 \mathrm{~s}[1.31,1.62])$ compared to when the right option was cued $(M=1.62[1.46,1.78]$. Similarly, when the right option was cued the right choice was faster $(M=1.44 \mathrm{~s}[1.28,1.60])$ compared to when the left option was cued $(M=1.55 s[1.39,1.71])$. Consistent with the observed choices, this congruence effect in RTs cues was larger in the preferential vs. perceptual frame as revealed by a 3way interaction between frame, cue, and choice $(b=0.13[0.03,0.22])$. See full parameter estimates in Table S2.

Information search. The choice and RT analyses suggest that relative value and cue had a differential impact on preferential vs. perceptual choice behavior. We reasoned that such difference could be accounted for by different information search patterns, given the free viewing nature of the task. Thus, we used eye fixation as a proxy for information search as participants sampled information from the two options. Average horizontal gaze trajectories are plotted time-locked to stimulus onset in Figure 4. The gaze trajectories exhibited several features of information search in our tasks. First, there was a prominent cueing effect such that on average, gaze first deviated toward the left location after a left cue and toward the right location after a right cue. In other words, participants tended to look at the cued option first in their information search, and this was true in both decision frames. This observation suggests that the peripheral cue - although entirely task-irrelevant and brief - attracted the initial fixation. Second, after the initial deviation, the gaze trajectories reversed their direction, suggesting that participants tended to inspect the other option after first inspecting the cued option. Third, the degree of the shift between the left and right option, or the amplitude of the oscillatory timecourse of gaze trajectory, appeared to be larger in the perceptual than the preferential frame. Fourth, there was also a leftward bias such that the leftward deviation was larger overall than the rightward deviation. This can also be seen in the center cue condition, which showed that the first deviation on average is toward the left option, even though the cue was located in the center. Such leftward bias in initial fixation has been reported in previous free viewing tasks (Foulsham \& Kingstone, 


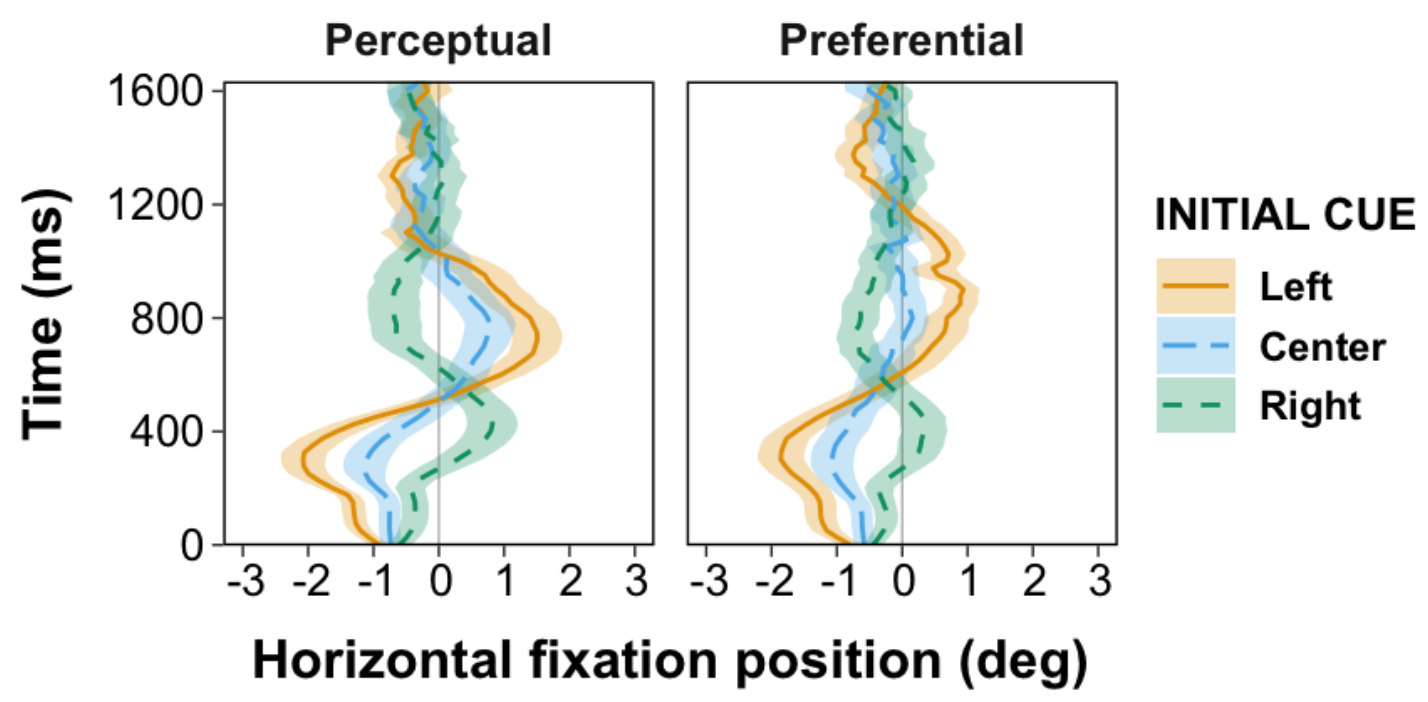

Figure 4. Average horizontal gaze trajectories time-locked to stimulus onset. Negative values indicate the left side of the screen, and positive values indicate the right side of the screen. The trajectories are separated by decision frame (panels), cue location (colors), and collapsed across other trial attributes (i.e., relative value and participants' choice). The error regions indicate standard errors of the average horizontal positions at that time point across participants.

2010; Foulsham et al., 2013). Indeed, there is a well-documented, small but consistent leftward bias in many visuospatial tasks (Jewell \& McCourt, 2000), which might result from reading habits and/or brain lateralization of attentional control (Rinaldi et al., 2014; Thut et al., 2006; Mesulam, 1981). This overall bias is orthogonal to our main experimental manipulations and as such, we will not consider it further in this report. In the following, we quantify three measures from the gaze trajectory data: gaze variability, relative dwell time, and gaze cascade, to examine how our experimental manipulations impacted information search.

Gaze variability. The first measure of information search behavior is how the degree to which gaze alternated between the two options. We calculated each trial's standard deviation in the horizontal eye position as a proxy for the magnitude of gaze alternation. A larger left-right shift pattern should give rise to a larger standard deviation in gaze position, potentially indicating a more balanced information search between left and right options. For example, if participants spend all their time at one location, the standard deviation is zero, whereas if they divide time equally, the standard deviation is maximal. Figure 5 shows that gaze variability decreased as the unsigned relative value increased $(b=-0.21[-0.27,-0.16])$. This effect of the unsigned relative value depended on the decision frame $(b=0.08[0.002,17])$, with gaze variability in the perceptual frame $(b=-0.19[-0.22,-0.15])$ being more sensitive to unsigned relative value than that in the preferential frame $(b=-0.11[-0.14,-0.07])$. Gaze variability was also reduced when cueing to the right vs. left $(b=-0.16[-0.26,-0.07])$, with this difference between cues being 


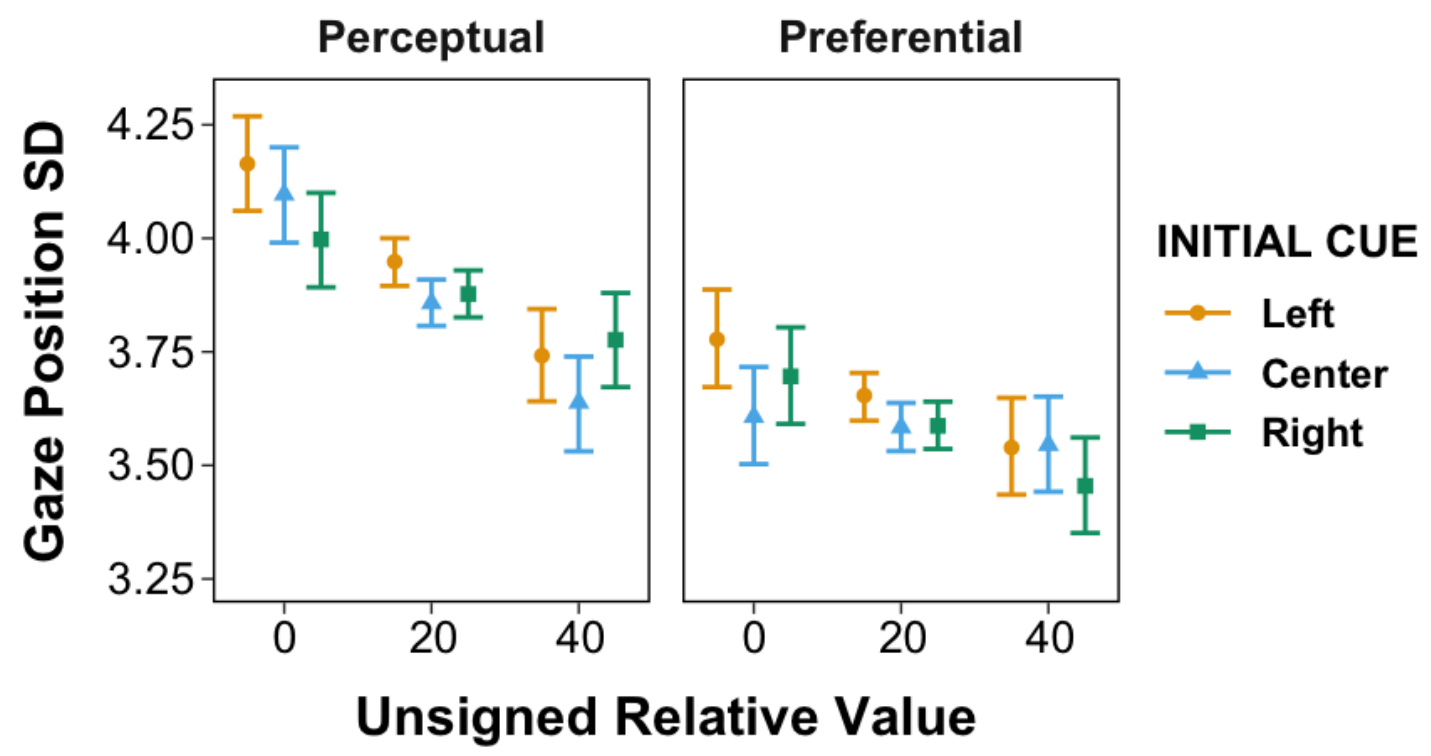

Figure 5. The posterior predicted means of gaze position standard deviation are plotted against the unsigned relative value (absolute difference in mean dots between options). The plots are conditioned by the task frame (panels) and cue location (colors). Error bars indicate $95 \%$ HDIs of the posterior predicted means.

reduced with larger unsigned relative values $(b=0.10[0.02,0.18])$. This latter effect likely reflects the overall leftward bias in gaze and will not be discussed further. Altogether the changes in gaze variability suggest that search was less variable (and less balanced) in the preferential frame and this difference between decision frames was more pronounced when the options were more similar.

Relative dwell time. For the second measure, we quantified the cue's overall impact on gaze by calculating the relative dwell time within a trial - the time participants spent looking at one option normalized by the total amount of time they spent looking at both options. Figure 6 plots the relative dwell time as a function of the unsigned relative value for the different cue types and decision frame. This analysis revealed that cueing the right option credibly increased the gaze dwell time on the right option $(b=0.48[0.17,0.79])$. Moreover, there was a credible interaction between the decision frame and the cue $(b=0.76[0.31,1.21])$ such that the effect of the cue was larger in the preferential frame $(b=1.07[0.91,1.23])$ as compared to the perceptual frame $(b=0.60[0.45,0.75])$. There was no credible effect of the unsigned relative value on dwell time. These results suggest that the cue, on average, attracted the first fixation to the cued option and led the eye to fixate on the cued option for longer. These cueing effects are more pronounced for the preferential frame than the perceptual frame.

Gaze cascade. As a final step in our analysis of information search, we examined the gaze cascade effect (Shimojo et al., 2003; Simion \& Shimojo, 2006, 2007). According to the gaze cascade effect, participants grow more likely to fixate on the item they are about to choose. Figure 7 plots the probability of fixating on the eventually chosen option 


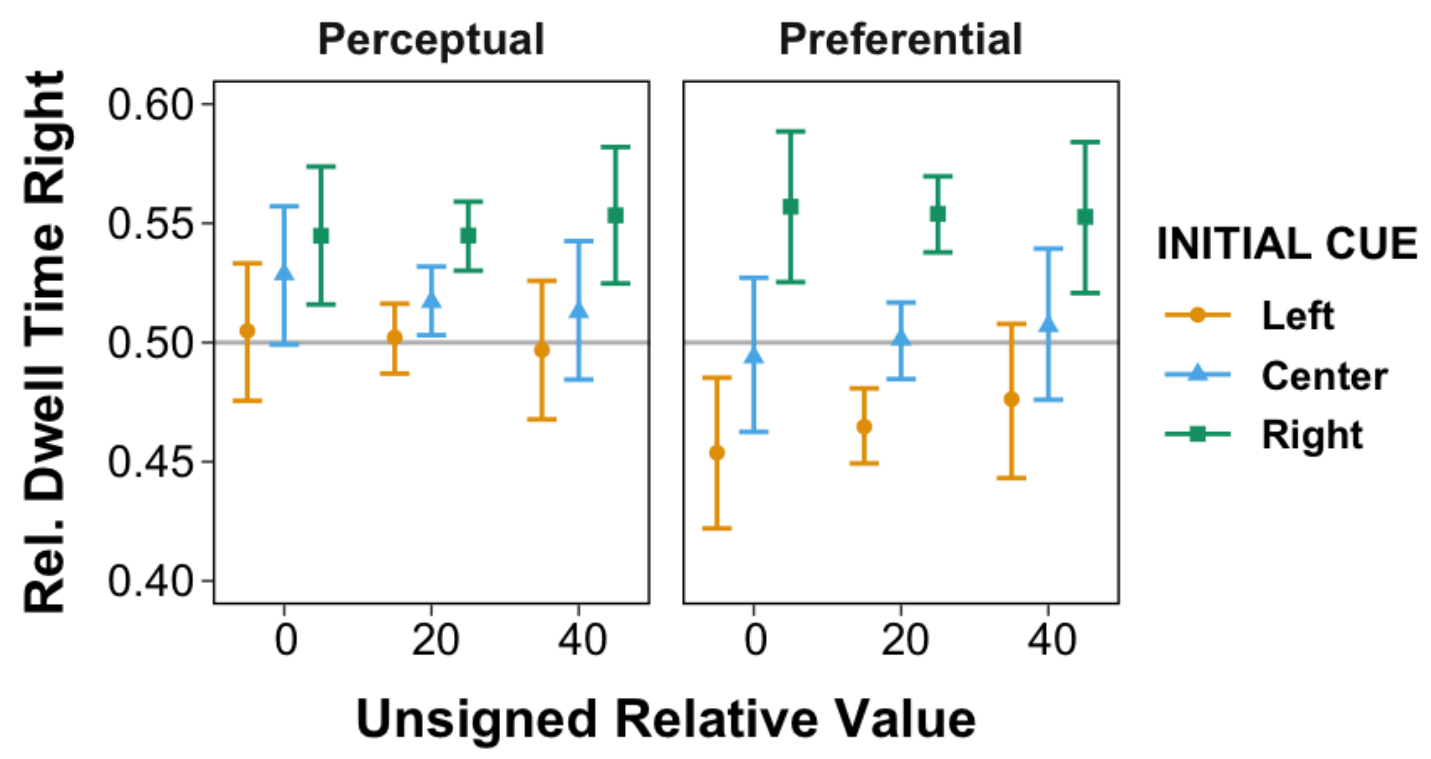

Figure 6 . The posterior predicted means of relative dwell time for the right option are plotted against the unsigned relative value (absolute difference in mean dots between options), conditioned by the decision frame (panels) and cue location (colors). Error bars indicate $95 \%$ HDIs of the posterior predicted means.

at different time points before the choice. As the plot shows, the likelihood of fixating on the chosen option increased until the choice was recorded. Figure 7 also suggests that this gaze cascade effect depended on the decision frame and attentional cue. A hierarchical logistic regression on whether the chosen option was fixated on (in the time window from 0 to $-250 \mathrm{~ms}$ ) with decision frame, cue location, and unsigned relative value as predictors supported this inference. In the preferential frame, participants were more likely to fixate on the chosen option than those in the perceptual frame $(b=0.44[0.08,0.80])$. There were also some credible effects of cue and unsigned relative value, but these are inconsistent thus we do not interpret them further (see Supplementary Table S6 and Figure S6).

\section{Summary}

We found that the preferential decision was impacted more by the exogenous attentional cue than the perceptual decision. Specifically, a greater value difference between the right and left option was needed for participants to choose the right option when the left option was cued (and vice versa when the right option was cued), and this difference was greater in the preferential frame than the perceptual frame. The attentional cue's differential effect also manifested during information search. The relative dwell time on a particular option was influenced to a greater extent by the cue in the preferential frame than the perceptual frame. We suggest the attentional cue's effect on preferential choice over and above its effect on perceptual choice is consistent with the AIV hypothesis, whereby attention to an option enhances its value and makes it more likely to be chosen.

Further support for the AIV hypothesis also comes from information search behavior 


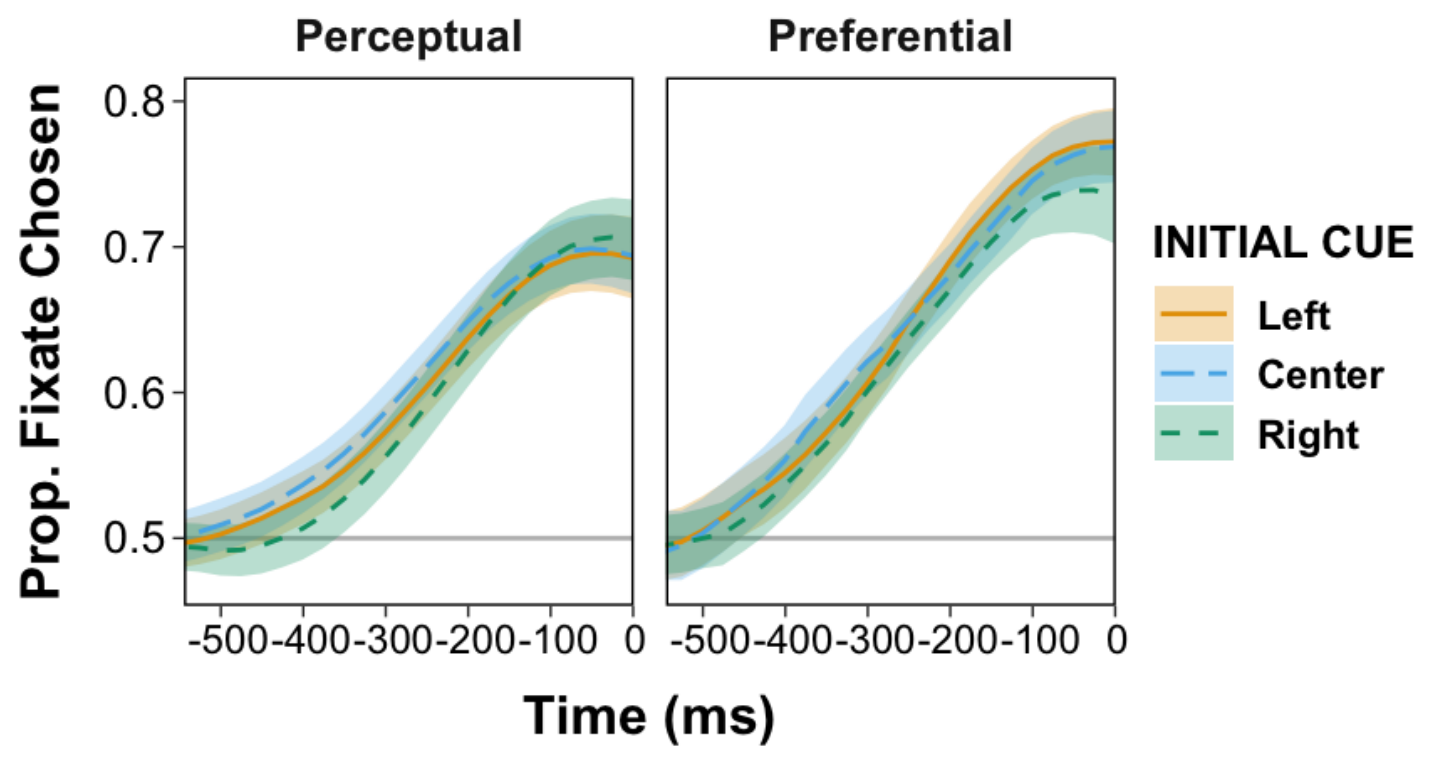

Figure 7. The probability of fixating on the eventually chosen option is time-locked to choice response up to $500 \mathrm{~ms}$ before the choice. The data was conditioned by the decision frame (panels) and cue location (color). Error regions indicate standard errors of the probabilities. Curves were smoothed using a 7-point rolling average.

late in the trial. In this case, a gaze cascade effect was observed where participants grew more likely to look at the item they were about to choose, and this effect was stronger in the preferential frame. Why does this differential gaze cascade effect support the AIV hypothesis? As Mullett \& Stewart (2016) have shown, a gaze cascade effect can emerge from an evidence accumulation decision process, where to make a decision, participants accumulate evidence for either option up to a threshold and then respond accordingly. But, to create a gaze cascade effect, the evidence accumulation process needs two properties: (1) a relative stopping rule where the decision to stop is based on the relative evidence for one option over the other(s); and (2) the evidence being accumulated is weighted more heavily in favor of the currently attended item. Thus, the larger gaze cascade effect in the preferential choice than the perceptual analog is consistent with an evidence accumulation process underlying both decisions. The AIV hypothesis implies a boost in the value of the attended option and hence the accumulated evidence for the option. Later, we use computational modeling to quantitatively assess how well an evidence accumulation process can explain our data, and if a greater weight is given to the attended option, particularly during preferential choice. Independent of the modeling results, however, the eye movement data support the AIV hypothesis with the attentional cue having a greater impact on search early in the time course of the decision and a larger gaze cascade effect later in the time course. Furthermore, across the time course, we see the consequences of these effects in the reduced variability in information search.

The potential difference in sensitivity and response times between the preferential and perceptual frames do identify some potential limitations for Study 1 and suggests an 
alternative explanation for at least the observed larger cueing effect in the preferential frame. For instance, lower sensitivity to the relative value could indicate more uncertainty such that any additional piece of information could have a greater effect on choice. Thus, the greater impact of the attentional cue could be due to the greater uncertainty in the preferential frame. The response time differences raise a similar alternative explanation. Follow-up analyses, however, revealed that these condition-level differences were largely driven by a few participants in the preferential frame who exhibited less sensitivity and had faster responses (see Supplementary Material 1.3.1). Furthermore, post-hoc matching analyses that equated sensitivity and response time across decision frames replicated the cueing effect, implying potential differences in levels of information processing cannot explain this cueing effect (see Supplementary Material 1.3.2).

Before describing our computational modeling results in which we further explore the linkage between attention and valuation, we conducted an additional study to provide converging evidence for the AIV hypothesis. In Study 2, we exerted more control on participants' information search pattern, thus controlling for the differences in sensitivity and response times between the decision frames. In addition, there were also differences in eye movements between the decision frames in Study 1. Past work has suggested that the apparent effect of attention on value is due to the sensorimotor aspect of eye movement and the mind aligning preferences in concordance with the motor movement (e.g., Shimojo et al., 2003; Simion \& Shimojo, 2007)(though see Nittono \& Wada, 2009; Bird et al., 2012). In Study 2, we addressed these issues using a fixed viewing study, eliminating differences in eye movement behavior between task frames. We also employed an interrogation protocol where we cued participants when to make a choice. Our goal was to equate motor movements, sensitivity and response times between task frames and thus conduct a more complete assessment of the AIV hypothesis a priori, while controlling for potential differences in information processing between the decision frames.

\section{Study 2: Fixed viewing study}

In this study, we changed the display so that the two options were presented one at a time in the center of the screen. We manipulated the duration of each presented option, thus controlling the time participants attended to each option without the need to move their eyes. Such a fixed viewing paradigm eliminated any difference in response times and eye movement patterns between decision frames. Although changing presentation duration is not a typical attention manipulation in laboratory studies, people tend to look at objects that interest them for a longer period in naturalistic viewing conditions (Henderson, 2003). Thus, we can use presentation duration as a proxy for attention. Importantly, the same duration manipulation was applied to the perceptual and preferential decision frames, allowing us to isolate the effect of our manipulation on valuation beyond its effect on perception. With this change in the method of manipulating attention, this study provided an opportunity to examine the generalizability of the observed differential attentional effects between the two decision frames. Furthermore, the study allowed us to assess whether the effect of attention on preference required eye movement per se, as suggested by past work (Shimojo et al., 2003; Simion \& Shimojo, 2007). We did not preregister this study. 


\section{Method}

Participants. In total, 63 (31 preferential and 32 perceptual) undergraduate participants from the Michigan State University Psychology subject pool took part in the study. In addition to receiving course credit for participating, they also earned a \$1-5 bonus based on their task performance. Michigan State University's Institutional Review Board approved the study.

Design. The study had a 2 (frame) $\times 5$ (stimulus duration $) \times 5$ (relative value) mixed design. The frame (preferential vs. perceptual) varied between participants and the other two factors varied within participants. The relative value was the difference in the mean number of dots between options (first minus second option: -50, -25, 0, 25, 50), made up of six combinations of option pairs. Stimulus duration was manipulated such that the first option was visible $33 \%, 50 \%, 67 \%$, or $75 \%$ of the total presentation time, over eight different presentation sequences (see Supplementary Material, Table S7).

Flash Stimulus. The flash stimulus was identical to that in Study 1 with the following modifications. First, the options were presented sequentially in the center of a computer screen. Second, we presented the two stimuli in either red or blue so that participants could easily differentiate between the two options. The brightness of the two colors was roughly equated in a pilot test by taking the average values of four test participants who completed a psychophysical isoluminance procedure using heterochromatic flicker photometry (Kaiser, 1991). The order of colors (blue or red option first) was counterbalanced across trials. Third, the number of dots in each option was generated from a Gaussian distribution truncated at 0 with a mean of 105, 130, or 155 dots and a standard deviation of 20 dot. We increased the range of mean values from Study 1 because pilot testing revealed that the sequential presentation reduced discriminability slightly.

Procedure. The study was held over a single 2-hour session. Similar to Study 1, participants were randomly assigned to either the preference or perceptual frame and received instructions for the flash task with two central stimuli: a red pond and a blue pond. The instructions were similar to Study 1. In the perceptual frame, participants were asked to choose if the red or blue pond had more fish on average, while in the preference frame, participants were asked to choose if they preferred to fish from the red or blue pond.

In a trial, after displaying a central fixation dot for $500 \mathrm{~ms}$, the flash stimuli (the two colored ponds) appeared in the center of the screen in a sequential, alternating fashion (Figure 8). For example, participants would first view a stream of blue dots updated every $50 \mathrm{~ms}$, followed by a stream of red dots at the same update rate and, on occasion, a second stream of blue dots. The color assigned to the first or second stimulus was randomized between participants. They were told that the dots represented blue or red fish from two ponds, so they could consider all blue fish, even if they re-appeared later, to be from the same pond. Participants were also instructed to wait until all the fish were presented and that they should immediately make their choice once the fish disappeared and were replaced by a central fixation point. The fixation point remained on the screen until participants pressed a key (labeled in red or blue) to indicate their choice. Similar to Study 1, participants earned points by catching fish, which was displayed as feedback at the end of the trial. The points were aggregated and scaled to generate a $\$ 1-5$ performance bonus paid at the end of the session. Participants in the perceptual frame $(M=\$ 4.92, S D=0.15)$ earned 


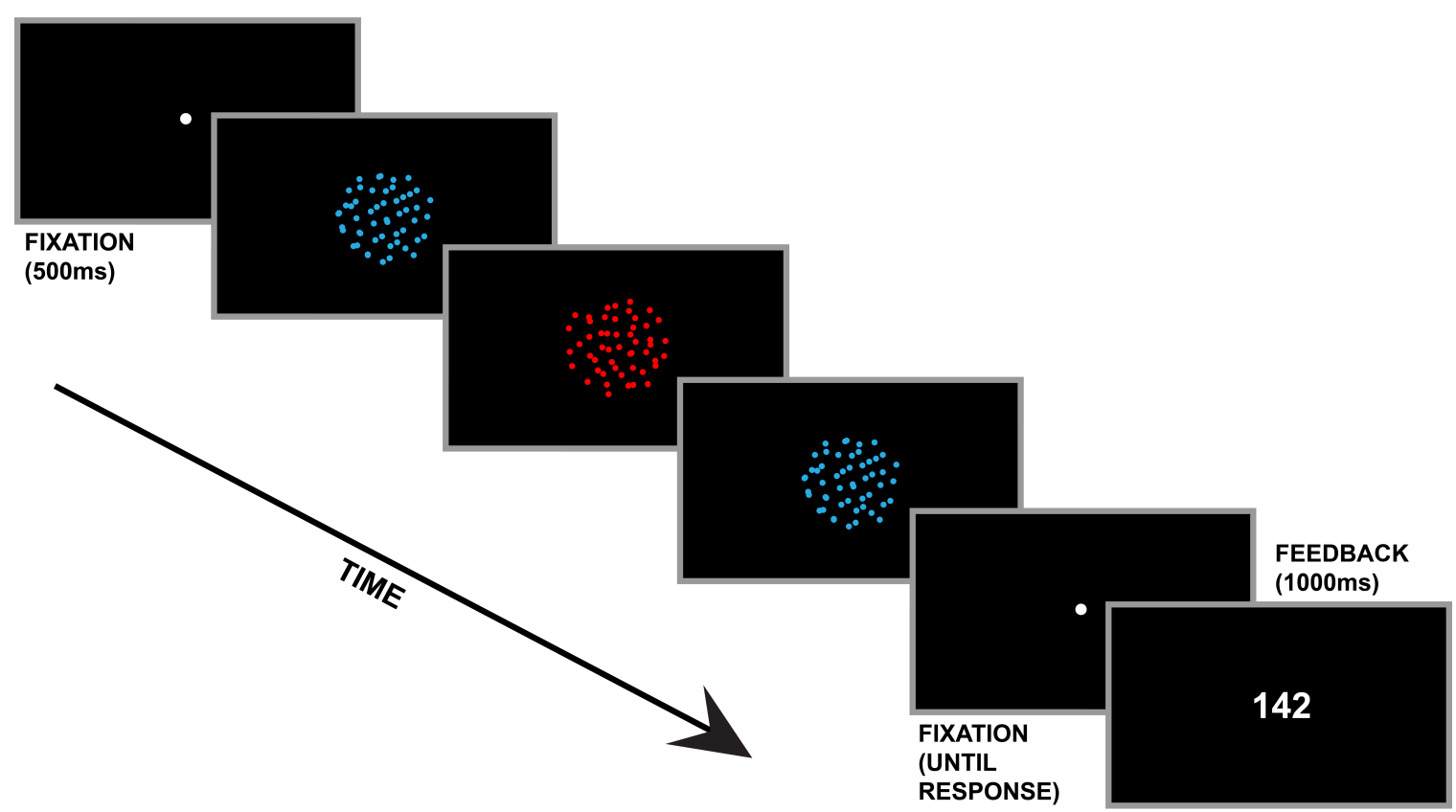

Figure 8. Trial schematic for Study 2. After a central fixation, the two stimuli were presented in an alternating sequence. The stimuli were presented either with one switch (blue-red, or red-blue, not shown) or two switches (blue-red-blue, as shown here, or redblue-red, not shown). Each stimulus was presented for a specific duration (see Supplemental Table S7). A central fixation point then appeared, prompting participants to respond. After the response, an appropriate feedback message was provided for each decision frame.

largely the same compensation compared to those in the preferential frame $(M=\$ 4.94$, $\left.S D=0.10) ; M_{D}=-0.02[-0.08,0.05]\right)$

As with Study 1, we removed trials where participants selected an incorrect button (chose neither the first nor the second option). Consistent with our previous work with the FGT (Pleskac et al., 2019), we also removed trials with responses faster than $0.25 \mathrm{~s}$. Responses faster than this timing threshold show no sensitivity to the relative value and were classified as guesses. The average number of removed trials under these cutoffs was $1.1(0.12 \%$ total $)(\mathrm{SD}=2.2)$ per participant. To minimize the chances that we included trials where participants were distracted, we also removed trials with response times longer than 5 seconds from all analyses. The average number of trials that exceeded the 5 s cutoff was $3.9(0.44 \%$ total $)(\mathrm{SD}=6.2)$ per participant.

\section{Results}

Choice behavior. Group-level psychometric functions show choice behavior for each condition in Figure 9. The functions show that participants were likelier to choose the first option when it had more dots on average. The psychometric function was again modeled with a hierarchical four-parameter logistic function where the probability of choosing the first option was a function of the relative value (First - Second). Similar to Study 1, we allowed the threshold $\mu$ and slope $\theta$ parameters to vary between the decision frame and relative duration of the first option. The parameters controlling the asymptote location 


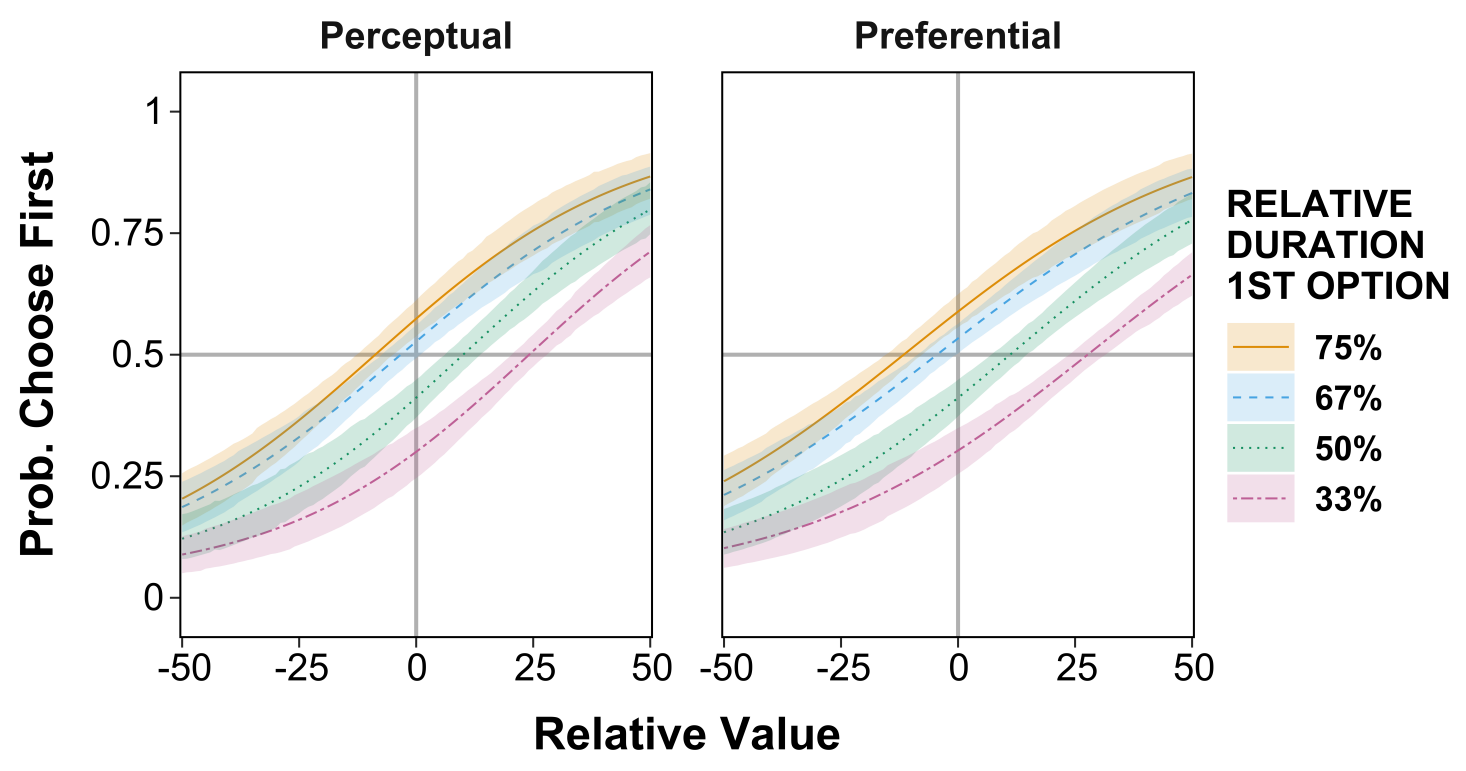

Figure 9. The probability of choosing the first option is plotted against the relative value (First - Second). The data was conditioned by the decision frame (panels) and relative duration of the first option (colors). The solid-colored dots represent the predicted choice proportions with the error regions indicating the $95 \%$ HDIs of the proportions.

and indexing the base rate of responding $(\gamma$ and $\lambda)$ were allowed to vary between decision frames but fixed between the duration manipulations (for a given decision frame). We again examined two aspects of these psychometric functions: thresholds and sensitivity (for group level estimate and comparisons, see Supplemental Table S8).

Threshold. Similar to the free-viewing study, we found that participants were more likely to choose the option that was presented for a longer relative duration, manifested as a horizontal shift in the psychometric function. For each step of change in the relative duration there was on average an increase of 20.40 dots $[5.45,39.31]$ in the threshold, $\mu$. Like the cue-based manipulation in Study 1, the degree of shift in the threshold depended on the decision frame. For instance, comparing the lowest (33\%) and highest (75\%) duration results in a credible shift in the threshold in the preferential frame $(M=39.31[36.17,42.67])$ and in the perceptual frame $(M=32.89[30.06,35.46])(M=6.42[2.24,10.68])$. Across all changes in relative duration, this shift in threshold was greater in the preferential frame, indicating that viewing duration had a greater effect on choice in the preferential frame $(M=3.51[1.07,5.84])$.

Sensitivity. In terms of sensitivity, overall, the psychometric function slopes were shallower compared to the free-viewing study. This decrease in sensitivity was likely due to a more difficult task when the two options are sequentially presented, which requires more memory and integration between stimulus presentations. In contrast to the free-viewing study, the sensitivity in terms of the slope of the psychometric functions at the thresholds were not credibly different between decision frames $(M=-0.0009[-0.0038,0.0018])$, suggesting that with controlled viewing we removed differential sensitivity in discriminating 
between the choice options across decision frames (see Supplemental Table S8). ${ }^{45}$

\section{Summary}

In Study 2, we directly controlled the viewing duration while presenting the two options sequentially at fixation. Three major findings emerged in this controlled viewing task. First, the effect of stimulus duration on choice was larger in the preferential decision frame as captured by shifts in the choice threshold, suggesting that while attention can modulate perception (Carrasco \& Barbot, 2019), it has a further and specific impact on an option's value representation. Second, there was no difference in the sensitivity to relative value between decision frames. Moreover, as response times were controlled experimentally, there were no differences in response times between decision frames. These results help rule out possible differences in levels of information processing as being responsible for the greater effect of attention on choice thresholds in the preferential frame compared to the perceptual frame. Third, as the relative duration of an option increased, the likelihood of choosing it increased. This result is consistent with other similar studies (Bird et al., 2012; Nittono \& Wada, 2009), implying that eye movement per se is not necessary for attention to exert an effect on choice. Altogether these results support the AIV hypothesis that paying attention to an option, manifested in a longer looking time, does lead to liking by enhancing stimulus value. To provide further support for this claim, we employed computational models to help isolate how attention impacted the underlying decision process.

\section{Modeling the effect of attention on evidence accumulation}

We used a diffusion decision model (DDM; Ratcliff et al., 2016; Busemeyer et al., 2019 ) to isolate how attention impacted preferences. A DDM models decision making as a sequential sampling process, where participants sequentially sample information about the options and accumulate the information as evidence to make a choice. The rate of evidence accumulation, $\delta$, captures the direction and speed at which evidence accumulates in the model. There is a starting point of the evidence, which captures an initial bias towards one option or the other. During an optional stopping procedure as in Study 1, where the time at which a choice is made is determined endogenously, DDM assumes that the choice is made when the quantity of accrued evidence reaches a pre-determined threshold. The location of the upper threshold is specified by the threshold separation parameter $\alpha$, with the bottom threshold located at 0 . During an interrogation protocol where the time of the choice is externally determined, as in Study 2, the location of the evidence is compared to a criterion and a choice is made accordingly.

This general evidence accumulation process provides a good account of both perceptual (e.g., Ratcliff \& Smith, 2004; Ratcliff et al., 2016) and preferential (e.g., Busemeyer \& Diederich, 2002; Busemeyer et al., 2019) decisions (Pleskac et al., 2019; Summerfield \&

\footnotetext{
${ }^{4} \mathrm{We}$ are not presenting response time results as the task used a cued response. Thus, the recorded response time is less meaningful.

${ }^{5}$ We also found evidence of a small recency effect in that participants slightly preferred to choose the most recently viewed option. In the one-switch condition, The posterior predicted likelihood of choosing the first option in this condition was $0.47[0.45,0.50]$ in the preferential frame and $0.48[0.46,0.50]$ in the perceptual condition. But, there was no difference between decision frames in this order effect. See Supplementary Material for more details.
} 
Tsetsos, 2012). However, the data from both the free and fixed viewing studies suggest that while attention impacts both choices, there are also differences in how it impacts choice in each decision frame. To examine the role of attention in these two decision frames, we formalized the DDM in terms of how eye gaze influences the evidence accumulation process (Krajbich et al., 2010; Krajbich \& Rangel, 2011; Smith \& Krajbich, 2019). According to these models, the rate of evidence accumulation is a function of the difference in the values of the two options. The value of the non-fixated option is discounted during evidence accumulation. Formally, the value of each option is specified by the mean number of dots, $\mu$, in each option so that

$$
\delta=c\left(\mu_{\text {fixated }}-\theta \times \mu_{\text {nonfixated }}\right)+\epsilon .
$$

Where the parameter $0<\theta<1$ discounts the value of the non-fixated option, and $c$ is a scaling constant. Following Cavanagh et al. (2014), this hypothesis can be implemented as a linear model, so that the drift rate is,

$$
\delta=\nu_{0}+\nu_{1} \times\left(\text { gaze }_{\text {right }} \times \mu_{\text {right }}-\text { gaze }_{\text {left }} \times \mu_{\text {left }}\right)+\nu_{2} \times\left(\text { gaze }_{\text {left }} \times \mu_{\text {right }}-\text { gaze }_{\text {right }} \times \mu_{\text {left }}\right)+\epsilon .
$$

Formulated this way, positive values of $\delta(\delta>0)$ indicate evidence of accumulation toward the right (or the first) option, and negative values indicate accumulation toward the left (or the second) option. The parameter $\nu_{0}$ is the baseline drift, and $\nu_{1}$ and $\nu_{2}$ determine the contribution of the fixated and non-fixated options, respectively. The gaze variables specify the relative amount of fixation time for each option during a trial - these are measured by eye tracking in Study 1 and by the duration of presentation in Study 2. The relationship between Equation 3 and Equation 2 can be seen by conditionalizing on when the right or left option is fixated on. For instance, if the right option is fixated on $\left(\right.$ gaze $_{\text {right }}=1$, gaze left $_{1}=0$ ), then Equation 3 can be written as $\nu_{1} \times \mu_{\text {right }}-\nu_{2} \times \mu_{\text {left }}$ where $\nu_{1}=c$ and $\nu_{2}=c \theta$. Thus, the degree to which the non-fixated option is discounted is the ratio of $\nu_{1}$ and $\nu_{2}$,

$$
\theta=\nu_{2} / \nu_{1}
$$

Equations 2 and 3 captures one way to model the AIV hypothesis with attention enhancing the value of an option (via discounting of the unattended options). However, another way attention could induce value is that attention could simply lead to additional information to be accumulated independent of the value of the options (Cavanagh et al., 2014). Therefore, we also included an additive gaze component to the drift rate model, so that,

$$
\begin{aligned}
\delta= & \nu_{0}+ \\
& \nu_{1} \times\left(\text { gaze }_{\text {right }} \times \mu_{\text {right }}-\text { gaze }_{\text {left }} \times \mu_{\text {left }}\right)+ \\
& \nu_{2} \times\left(\text { gaze }_{\text {left }} \times \mu_{\text {right }}-\text { gaze }_{\text {right }} \times \mu_{\text {left }}\right)+ \\
& \nu_{3} \times\left(\text { gaze }_{\text {right }}-\text { gaze }_{\text {left }}\right)+\epsilon .
\end{aligned}
$$

The drift rate coefficients $\nu$ help determine how attention induces value. The parameters $\nu_{1}$ and $\nu_{2}$ determine how much the accumulated value is enhanced by attending to an option. The parameter $\nu_{3}$ determines the weight given to the independent contribution of gaze to 
Table 1

Mean and 95\% HDI posterior estimates of the group-level parameters from attention-based DDM for the free-viewing Study 1.

\begin{tabular}{rccc}
\hline & Preference & Perceptual & Preference vs. Perceptual \\
\hline$\nu_{0}$ (Baseline drift) & $0.014[-0.099,0.121]$ & $0.035[-0.097,0.159]$ & $-0.021[-0.186,0.152]$ \\
$\nu_{1}$ (Fixated option) & $2.44[2.393,2.487]$ & $2.553[2.515,2.592]$ & $\mathbf{- 0 . 1 1 4}[\mathbf{- 0 . 1 7 5 , - 0 . 0 5 3}]$ \\
$\nu_{2}$ (Nonfixated option) & $1.518[1.423,1.611]$ & $2.079[2.012,2.145]$ & $\mathbf{- 0 . 5 6 1}[\mathbf{- 0 . 6 7 5 , - 0 . 4 4 2}]$ \\
$\nu_{3}$ (Additive contribution of gaze) & $0.594[0.413,0.767]$ & $0.392[0.21,0.574]$ & $0.202[-0.053,0.456]$ \\
$\eta$ (Between-trial drift variability) & $0.321[0.121,0.518]$ & $0.356[0.158,0.555]$ & $-0.036[-0.328,0.233]$ \\
$\beta_{\text {left }}$ (Relative start point for left cue) & $0.419[0.23,0.614]$ & $0.448[0.256,0.648]$ & $-0.029[-0.313,0.245]$ \\
$\beta_{\text {center }}$ (Relative start point for center cue) & $0.571[0.363,0.775]$ & $0.596[0.385,0.796]$ & $-0.025[-0.323,0.266]$ \\
$\beta_{\text {right }}($ Relative start point for right cue) & $0.73[0.549,0.894]$ & $0.695[0.504,0.87]$ & $0.035[-0.225,0.295]$ \\
$\gamma($ Between-trial start-point variability) & $0.122[0.1,0.158]$ & $0.122[0.1,0.158]$ & $0[-0.052,0.054]$ \\
$\alpha$ (Threshold separation) & $0.683[0.44,0.922]$ & $0.711[0.468,0.962]$ & $-0.028[-0.373,0.321]$ \\
NDT $^{\prime}$ (Relative nondecision time) & $0.599[0.566,0.633]$ & $0.599[0.565,0.631]$ & $0[-0.047,0.048]$ \\
\hline
\end{tabular}

Relative non-decision time is relative to the smallest response time observed.

the drift rate. If $\nu_{1}=\nu_{2}=0$ and $\nu_{3}>0$, then this reduces to a model where attention only induces additive value independent of the options' values. If $\nu_{1}=\nu_{2}>0$ and $\nu_{3}=0$, then this reduces to a model where attention induces value strictly by enhancing the value of the attended-to-option.

We fit this full DDM using Equation 5 to model the drift rate, to both decision frames in the free-viewing and fixed-viewing study. As the free-viewing study used an optional stopping procedure where the participant determined when to make a decision, we modeled this process as an accumulate-to-bounds DDM. Thus, the model predicts both the choice and response times. In contrast, the fixed-viewing study used an interrogation procedure where the experimental protocol determined when a decision was to be made. Therefore, the choices were modeled as a signal detection process where a person accumulates evidence and then examines if the evidence points to the first or second option when called to make a choice. For the free-viewing study, we used the proportion of time participants fixated on the right option relative to the sum of the time they fixated on either the left or right option to index relative gaze. For the fixed viewing study, we use the relative duration that each option was shown as a measure of relative gaze. The models were implemented within a Bayesian hierarchical structure modeling choices (and response times for the free-viewing study) at the individual participant level. ${ }^{6}$ For precise details, including model fits and model comparisons see the Supplementary Material (Supplementary Material 3).

\section{Study 1: Cued free-viewing}

We can use the parameters of the DDM to isolate different causes for the change in choice and RT among the cueing conditions (Table 1). One explanation is that the cue biased the starting point of evidence toward one choice or another. Indeed comparing the left to the right cue, there was a credible shift in the start point for the preference frame $(M=0.310[0.033,0.584])$ and a similar (but not credible) shift in the perceptual

\footnotetext{
${ }^{6}$ The supplementary material also reports model fits for models without the additive term (interactive model; Equation 2) and with only the additive term (additive model). A consistent winner of the model comparisons did not emerge so we report the full model (Equation 5) in the text as it is the most informative.
} 
frame $(M=0.247[-0.037,0.522])$. However, there is not a credible difference between the preference and perceptual frames in terms of the cue effect on the relative start point $(M=0.064[-0.334,0.459])$. This result would seem to rule out the explanation that the greater effect of the cue in the preferential frame was due to it having a greater impact on the initial start point of the evidence accumulation process.

A second means by which the attentional cue impacted choice is via the evidence being accumulated. Recall that the attentional cue also impacted what option participants fixated on and how long they fixated on it. The DDM reveals that this impact on information search behavior also shaped how evidence was accumulated. To see this, we calculated the ratio of the drift rate coefficients $\nu_{1}$ and $\nu_{2}$ to estimate the degree to which the non-fixated option was discounted ( $\theta$, see Equation 4). There was credible discounting in both the perceptual $(\theta=0.814[0.786,0.842])$ and preferential frames $(\theta=0.622[0.583,0.660])$. But, the degree of discounting was greater in the preferential frame $(-0.192[-0.242,-0.145])$. Thus, attention to one option meant that the information extracted from the unattended option was discounted relative to the attended option. This discounting happened to a greater extent in the preferential frame than the perceptual frame.

The DDM parameters offer two additional observations. First, the drift coefficients $\nu_{1}$ and $\nu_{2}$ in the preference frame were credibly lower than those in the perceptual frame, implying that for both fixated and nonfixated options, participants extracted less information from the options to make a decision. Second, for both decision frames $\nu_{3}$ was credible (but not credibly different between frames), indicating an additive contribution of gaze to the drift rate. ${ }^{7}$

These modeling results again raise the question of whether the impact of attention on valuation via the discounting of the unattended option was driven largely by how participants freely moved their eyes and allocated their attention to options as they made their choice. Therefore, we fit an equivalent model to the fixed-viewing study, which we turn to next.

\section{Study 2: Fixed-viewing}

During the fixed-viewing study, options were presented one at a time for different durations. If the differences between the perceptual and preference frame were largely due to differences in how participants voluntarily controlled their eye movement while searching for information, then they should largely vanish under this fixed viewing setting. However, we still observed a greater shift in the psychometric thresholds in the preference frame. The DDM reveals that this shift, similar to the free-viewing study, is due to a greater discounting of the non-fixated (not shown) option in the preference frame $(\theta=0.711[0.68,0.742])$ compared to the perceptual frame $\left(\right.$ thet $\left.a=0.76[0.73,0.791] ; M_{\text {diff }}=-0.049[-0.092,-0.005]\right)$

\footnotetext{
${ }^{7}$ Model comparisons reported in the supplementary material indicate, in fact, the perceptual frame is better modeled where value and gaze each have independent contributions to the drift rate, suggesting the discounting of the nonfixated option was marginal (though credible) in the perceptual frame. In contrast, a model that only included the interactive model (Equation 2) better modeled the preference frame indicating that in the preference frame, there was a greater discounting of non-fixated options.
} 
Table 2

Mean and 95\% HDI posterior estimates of the group-level parameters from attention-based DDM for the fixed-viewing Study 2.

\begin{tabular}{rccc}
\hline & Preference & Perceptual & Diff \\
\hline$\nu_{0}$ (Baseline drift) & $-3.739[-4.902,-2.525]$ & $-3.599[-4.942,-2.353]$ & $-0.140[-1.888,1.651]$ \\
$\nu_{1}$ (Fixated option) & $0.545[0.524,0.566]$ & $0.590[0.566,0.613]$ & $\mathbf{- 0 . 0 4 5}[\mathbf{- 0 . 0 7 7 , - 0 . 0 1 4}]$ \\
$\nu_{2}$ (Nonfixated option) & $0.387[0.3680 .407]$ & $0.449[0.426,0.472]$ & $\mathbf{- 0 . 0 6 1}[\mathbf{- 0 . 0 9 2 , - 0 . 0 3 2}]$ \\
$\sigma$ (Within-trial variability) & $0.062[-2.024,1.858]$ & $-0.102[-2.078,1.809]$ & $0.040[-2.690,2.787]$ \\
$\nu_{3}$ (Additive contribution of gaze) & $0.253[0,0.633]$ & $0.247[0,0.611]$ & $0.006[-0.524,0.561]$ \\
$\eta$ (Between-trial drift variability) & $0.016[0,0.047]$ & $0.01[0,0.028]$ & $0.007[-0.027,0.046]$ \\
\hline
\end{tabular}

The within-trial variability parameter measures the degree to which other factors entered influenced evidence accumulation while the between-trial drift variability measures how much the drift rate varied from trial to trial. The former proved necessary to include in modeling the choices from the fixed-viewing study.

(Table 2). ${ }^{89}$

Notably, the drift rate coefficients $\nu_{1}$ and $\nu_{2}$ were lower for both the perceptual and preferential frames in the fixed-viewing study compared to the free-viewing study. Moreover, there was less of a difference between the perceptual and preference frames in the fixed-viewing study. These differences between the studies explain why there was an overall lower sensitivity to the relative value in the fixed-viewing study and also a non-credible difference between decision frames (see Figures 2 and 9). Finally, in the fixed-viewing study, the additive contribution of gaze did not have a credible effect on the drift rate for both preference and perceptual frames. This difference between free and fixed viewing studies suggests that the free allocation of gaze and/or an optional stopping response process promotes a greater independent influence of the gaze on drift rate. Such a difference may help explain the different contributions of this independent factor across studies (see Cavanagh et al., 2014; Smith \& Krajbich, 2019).

\section{Summary}

The computational modeling of the decision process via the DDM helped reveal mechanisms through which attention impacts the evidence accumulation process. Most importantly, the models support the conclusion that the underlying mechanism for lookinginduced liking is via attention-enhancing option valuation. They showed that attending to an option enhances the value of the attended-to option and this attention-induced valuation has a greater impact during preferential choice. The computational modeling also helped further rule out several alternative explanations. There was no credible difference between

\footnotetext{
${ }^{8}$ In a second set of models, we allowed the relative duration of the stimuli to impact the start point of the evidence accumulation, analogous to how the attentional cue impacted the start point for the free-viewing study. However, this model provided a worse fit to the data, suggesting the relative duration primarily impacted the evidence accumulation. See supplementary material for further details.

${ }^{9}$ In a third set of models, we examined whether the sequential presentation impacted evidence accumulation because when the first option was presented, participants did not know the value of the second option. To reflect this, we parameterized a set of DDMs such that for the duration of the first presentation, the drift was solely determined by the value of the first option. Then for the second and third presentations, the drift was determined as specified in Equations 3 and 5. These models provided worse fits to the data than the ones presented here, suggesting the two options were compared to accumulate evidence, perhaps via a buffer of stored information.
} 
decision frames in terms of the relative start point and choice thresholds, thus ruling out an explanation of the greater effects of attention in the preferential frame as due to differences in levels of information processing between the decision frames. Moreover, as we observed the same effect of the attentional manipulations on enhancing option valuation with and without eye movements, we also can rule out the explanation that eye movement is the driving factor. Instead, the effect appears to be driven by the allocation of attention.

\section{General Discussion}

Do people grow to like what they attend to? Answering this question has important practical implications helping explain why products that attract more attention are more likely to be chosen (Chandon et al., 2009). It could also be used to help improve decisions such as in nudging healthy food choices (Hare et al., 2011; Leng et al., 2017). These behavior-level considerations aside, answering this question also requires us to investigate the fundamental role of attention in the construction of preference (Orquin \& Loose, 2013; Weber \& Johnson, 2009; Mormann \& Russo, 2021). By manipulating attention both with a peripheral cue and differential exposure of the potential options, in combination with eye tracking and computational modeling, our study provides converging evidence for the AIV hypothesis that attention increases the value of an option.

\section{Key findings and their relationship to existing work}

The support for the AIV hypothesis comes from several key findings. First, a taskirrelevant peripheral cue biased choice toward the cued option (Figure 2). The cue was designed to elicit an involuntary shift of attention and as such allowed us to manipulate attention in an unobtrusive way. Second, the cue influenced information search pattern during the trial, such that it shifted the initial fixation toward the cued location (Figure 4) and increased the overall dwell time on the cued option (Figure 6). These cueing effects were present in both the perceptual and preferential decision frames but were more pronounced for the preferential decision. Related to these findings, we also observed a stronger gaze variability and gaze cascade effect for preferential than perceptual decision frame (Figure 5 and 7). Third, our computational modeling analyses revealed that a DDM that incorporates visual attention can account for our behavioral data. In particular, the modeling results suggest that attention can have both additive and multiplicative effects on valuation and it enhances the value of the attended option.

While there is evidence consistent with the claim that people like what they look at (Armel et al., 2008; Milosavljevic et al., 2012; Pärnamets et al., 2015; Reeck et al., 2017; Smith \& Krajbich, 2019; Towal et al., 2013; Zoltak et al., 2018), there is also evidence that people look at what they like (Anderson et al., 2011; Anderson \& Yantis, 2013; Della Libera \& Chelazzi, 2009; Navalpakkam et al., 2010). In general, these two processes (looking induced liking and vice versa) can easily become intertwined in any task such that it is difficult to pinpoint the direction of causal influence. By manipulating attention on a trialby-trial basis, our study provides strong evidence for a critical component of attentionvaluation interaction - the AIV hypothesis.

Our results help untangle explanations of phenomena like the gaze-cascade effect.

One explanation of this effect that has been put forth is a self-reinforcing, positive feed- 
back loop, composed of looking induced liking and liking induced looking (Shimojo et al., 2003). However, as we discussed earlier, computational work suggests gaze cascade effect can emerge from an evidence accumulation model that uses a relative stopping rule where the decision to stop is based on the relative evidence for one option over the other(s), and more weight for evidence accumulated from the fixated option as in the AIV hypothesis (Mullett \& Stewart, 2016). Our experimental and modeling work supports the latter explanation. We showed that an evidence accumulation model gives a good account of both preferential and perceptual decisions. We also established both with behavioral analyses and computational modeling that there was discounting of the non-fixated option and that the discounting was greater in the preferential option. Consistent with these results we observed a gaze cascade in both the preferential and perceptual frames, but the effect was stronger in the preferential frame. Zooming out to the full time course of the decision process, this differential effect of attention on preferential decisions in both early and late time periods within a trial provide converging evidence of the unique contribution of attention on the value representation over and above its effects on perception.

In general, this dissociation between preference and perception is important because attention can shape early perception to enhance apparent salience so that an enhanced perceptual representation could lead to preference. Previous work has not considered this possibility and as such, their results in fact do not pinpoint a specific role of attention on valuation. By comparing the preferential and perceptual decision frames on the same stimulus display, our results demonstrated that attention had a larger impact on preferential than perceptual choice. Our results thus support the AIV hypothesis in showing a specific role of attention on valuation above and beyond its influence on perception.

This differential impact of attention on preferential and perceptual choice also speaks to the general question of the commonalities and differences between these two types of decisions (Dutilh \& Rieskamp, 2016; Pleskac et al., 2019; Summerfield \& Tsetsos, 2012; Zeigenfuse et al., 2014). A comprehensive comparison between these two types of decisions is beyond the scope of this paper. But, our results here support two general conclusions. The first conclusion is that a common decision process is used to make both preferential and perceptual decisions. This conclusion is supported by our finding that both decisions are well accounted for by a sequential sampling process where people accumulate information over time to determine a choice. But, this brings us to the second conclusion. According to our results, the properties of the information search and the accumulation process are somewhat different between the two types of decisions. Obviously, in many cases, the information can and will differ between these two types of decisions. But, here we show that even if we equate this information as best as possible we still observed differential effects of attention.

Nevertheless, we caution that there are limitations to this comparison between decision frames and more work is needed to isolate the causal factors that lead to these differences. One possible reason for this difference is that the feedback and payoff between the two frames are also different (Dutilh \& Rieskamp, 2016). In the preferential frame, the feedback and payoff was based on a single sample from an option, which captures a common property of preferential decisions where the outcome is uncertain (Luce \& Raiffa, 1957). In comparison, in the perceptual frame the feedback and payoff was based on the mean of the underlying distribution. Thus, a choice was either correct or incorrect (Hanks 
\& Summerfield, 2017). It is possible that some aspects of the feedback and payoff structure also help drive the process-level differences we have observed. As we outline later, a more compelling mechanistic explanation may rest in the additional valuation processes needed to make preferential decisions. We would also highlight that the difference between these two types of decisions is more a difference in degree. For instance, in the perceptual frame, participants are rewarded for accurate performance. This procedure is consistent with many perceptual decision making tasks, but it does mean that some value-based processing is being used. In a similar way, the feedback could be adjusted. For instance, a perceptual frame might ask participants to choose the option with the higher average reward or to identify the option that they predict will have the highest number of dots in the next draw. We suspect that changes that make one frame more similar to the other will also shape choice behavior to be more similar. Overall, we believe this direct comparison between preference and perceptual decision making is a fruitful comparison. After all, many advances in our understanding of preferential decision making have come by reasoning via analogy from perception (Kahneman, 2003) and vice versa (Masin et al., 2009).

Our results are also informative on whether motor movement per se plays a causal role in the effect of attention on preferential choice. Earlier studies found that without eye movement, there is a lack of exposure duration effect (Shimojo et al., 2003; Simion \& Shimojo, 2006), suggesting that eye movement prompts the decision maker to align their preferences to the motor movement itself (Bem, 1967; Fazio et al., 1977). However, this effect has been controversial as subsequent studies found that eye movement is not necessary for an exposure duration effect (Bird et al., 2012; Nittono \& Wada, 2009). Results from our Study 2 agree with these latter studies, and suggest that visual attention to an optionwhether it be due to an eye movement or not-results in magnifying the value of the option.

\section{Potential mechanisms of AIV}

What are the underlying mechanisms for the observed attention-induced valuation effect? Our computational modeling results provide some hints regarding possible mechanisms. The model assumes that people accumulate the differences in value between the two options in a sequential sampling process, and, critically, attention enhances the valuation of the attended option by discounting the unattended option. Our model also allowed an independent, additive, contribution of attention to valuation but its impact was not consistently observed-present in Study 1 but absent in Study 2. The presence of this independent contribution has been controversial (see Cavanagh et al., 2014; Smith \& Krajbich, 2019). Our results suggest that motor movement during free viewing might promote such an independent contribution. More relevant for the current discussion, regarding the difference between the two decision frames, the most consistent finding was a stronger discounting of the unattended option in the preferential than perceptual decision frame. Here, we propose that these effects can be understood within a computational framework based on normalization.

Broadly speaking, normalization is a process in which the mental representation of an item is scaled by its spatial and temporal neighbors, usually in a divisive manner such that the representations mutually inhibit each other. Although first proposed to explain neuronal activity in the primary visual cortex (Heeger, 1992), a normalization mechanism can explain behavioral and neural measurements across a wide range of sensory and cogni- 
tive domains, implying that normalization may be a canonical neural computation in the brain (reviewed in Carandini \& Heeger, 2012). Relevant to the current study, attentional effects in the visual cortex can also be modeled as modulating normalization such that the attended option receives a gain modulation which further inhibits the unattended option via normalization (J. Lee \& Maunsell, 2009; Reynolds \& Heeger, 2009). Thus, previous literature provides strong evidence for the role of normalization in perceptual processing and attentional modulation.

At the same time, context-dependent valuation among choice options is often observed and can be naturally explained by a framework of value normalization (reviewed in Louie \& Glimcher, 2012; Rangel \& Clithero, 2012). Indeed, neuroeconomic studies have found normalized neural representations of value in a number of key brain areas involved in decision making, such as the orbitofrontal cortex, anterior cingulate cortex, and posterior parietal cortex (Cai \& Padoa-Schioppa, 2012; Louie et al., 2011; Padoa-Schioppa, 2009). Since normalization essentially accentuates the difference among competing representations, our implementation of a discounting of the unattended item in the DDM may be thought of as a manifestation of normalization.

Given the normalization framework, why is there a stronger discounting (or normalization) in the preferential than the perceptual frame in our task? We speculate that this result is due to the ubiquitousness of normalization in the brain (Carandini \& Heeger, 2012). It is possible that attention may modulate both sensory normalization and value normalization. Under this scenario, perceptual decisions are influenced by sensory normalization, whereas preferential decisions are influenced by both sensory and value normalization (because it receives input from perceptual analysis). Thus, the latter would exhibit a larger attentional modulation. We note a few studies have reported that neural responses in several areas of the reward circuit are modulated by both option value and eye gaze (McGinty et al., 2016; Lim et al., 2011), potentially consistent with our conjecture of attention-modulated value normalization. However, these studies did not include a perceptual condition to isolate the effect of attention on valuation. Altogether we admit this normalization account is speculative, but we hope it will stimulate further research into the mechanisms of attentionvalue interaction. Certainly, more work is needed to disentangle the effect of attention on perception and its effect on valuation in both behavior and neural responses.

\section{Conclusion}

William James (1890/1950) once wrote, "Millions of items of the outward order are present to my senses which never properly enter into my experience. Why? Because they have no interest for me. My experience is what I agree to attend to. Only those items which I notice shape my mind - without selective interest, experience is an utter chaos. Interest alone gives accent and emphasis, light and shade, background and foreground intelligible perspective, in a word" (p. 168). Our results suggest that attention not only accents and emphasizes but also actively shapes the value people place on options as they construct a preference.

\section{References}

Anderson, B. A., Laurent, P. A., \& Yantis, S. (2011). Value-driven attentional capture. 
Proceedings of the National Academy of Sciences, 108(25), 10367-10371. doi: 10.1073/ pnas.1104047108

Anderson, B. A., \& Yantis, S. (2013). Persistence of value-driven attentional capture. Journal of Experimental Psychology: Human Perception and Performance, 39(1), 6. doi: $10.1037 / \mathrm{a} 0030860$

Armel, K. C., Beaumel, A., \& Rangel, A. (2008). Biasing simple choices by manipulating relative visual attention. Judgment and Decision making, 3(5), 396-403.

Bem, D. J. (1967). Self-perception: An alternative interpretation of cognitive dissonance phenomena. Psychological Review, 74(3), 183-200. doi: 10.1037/h0024835

Bird, G. D., Lauwereyns, J., \& Crawford, M. T. (2012). The role of eye movements in decision making and the prospect of exposure effects. Vision Research, 60, 16-21. doi: $10.1016 /$ j.visres.2012.02.014

Brainard, D. H. (1997). The psychophysics toolbox. Spatial vision, 10(4), 433-436.

Broadbent, D. (1958). Perception and communication.

Busemeyer, J. R., \& Diederich, A. (2002). Survey of decision field theory. Mathematical Social Sciences, 43(3), 345-370. doi: 10.1016/S0165-4896(02)00016-1

Busemeyer, J. R., Gluth, S., Rieskamp, J., \& Turner, B. M. (2019). Cognitive and neural bases of multi-attribute, multi-alternative, value-based decisions. Trends in Cognitive Sciences, 23(3), 251-263. doi: 10.1016/j.tics.2018.12.003

Cai, X., \& Padoa-Schioppa, C. (2012). Neuronal encoding of subjective value in dorsal and ventral anterior cingulate cortex. Journal of Neuroscience, 32(11), 3791-3808.

Carandini, M., \& Heeger, D. J. (2012). Normalization as a canonical neural computation. Nature Reviews Neuroscience, 13(1), 51-62.

Carrasco, M. (2011). Visual attention: The past 25 years. Vision research, 51(13), 14841525.

Carrasco, M., \& Barbot, A. (2019). Spatial attention alters visual appearance. Current Opinion in Psychology, 29, 56-64. doi: 10.1016/j.copsyc.2018.10.010

Carrasco, M., Ling, S., \& Read, S. (2004). Attention alters appearance. Nature Neuroscience, 7 (3), 308-313. doi: http://doi.org/10.1038/nn1194

Cavanagh, J. F., Wiecki, T. V., Kochar, A., \& Frank, M. J. (2014). Eye tracking and pupillometry are indicators of dissociable latent decision processes. Journal of Experimental Psychology: General, 143(4), 1476-1488. doi: 10.1037/a0035813

Chandon, P., Hutchinson, J. W., Bradlow, E. T., \& Young, S. H. (2009). Does in-store marketing work? effects of the number and position of shelf facings on brand attention and evaluation at the point of purchase. Journal of Marketing, 73(6), 1-17. doi: 10.1509/ jmkg.73.6.1 
Curcio, C. A., Sloan, K. R., Kalina, R. E., \& Hendrickson, A. E. (1990). Human photoreceptor topography. Journal of Comparative Neurology, 292(4), 497-523.

Della Libera, C., \& Chelazzi, L. (2009). Learning to attend and to ignore is a matter of gains and losses. Psychological Science, 20(6), 778-784. doi: 10.1111/j.1467-9280.2009.02360.x

Desimone, R., \& Duncan, J. (1995). Neural mechanisms of selective visual attention. Annual review of neuroscience, 18(1), 193-222.

Dutilh, G., \& Rieskamp, J. (2016). Comparing perceptual and preferential decision making. Psychonomic Bulletin \& Review, 23(3), 723-737. doi: 10.3758/s13423-015-0941-1

Everling, S., Tinsley, C. J., Gaffan, D., \& Duncan, J. (2002). Filtering of neural signals by focused attention in the monkey prefrontal cortex. Nature neuroscience, 5(7), 671-676.

Fazio, R. H., Zanna, M. P., \& Cooper, J. (1977). Dissonance and self-perception: An integrative view of each theory's proper domain of application. Journal of Experimental Social Psychology, 13(5), 464-479. doi: 10.1016/0022-1031(77)90031-2

Foulsham, T., Gray, A., Nasiopoulos, E., \& Kingstone, A. (2013). Leftward biases in picture scanning and line bisection: A gaze-contingent window study. Vision research, $78,14-25$.

Foulsham, T., \& Kingstone, A. (2010). Asymmetries in the direction of saccades during perception of scenes and fractals: Effects of image type and image features. Vision research, 50(8), 779-795.

Fuller, S., \& Carrasco, M. (2006). Exogenous attention and color perception: Performance and appearance of saturation and hue. Vision Research, 46(23), 4032-4047. doi: 10.1016/ j.visres.2006.07.014

Gelman, A., Carlin, J. B., Stern, H. S., Dunson, D. B., Vehtari, A., \& Rubin, D. B. (2013). Bayesian data analysis. CRC press.

Ghaffari, M., \& Fiedler, S. (2018). The power of attention: Using eye gaze to predict otherregarding and moral choices. Psychological science, 29(11), 1878-1889. doi: 10.1177/ 0956797618799301

Goodrich, B., Gabry, J., Ali, I., \& Brilleman, S. (2020). rstanarm: Bayesian applied regression modeling via Stan. Retrieved from https://mc-stan.org/rstanarm (R package version 2.21.1)

Hanks, T. D., \& Summerfield, C. (2017). Perceptual decision making in rodents, monkeys, and humans. Neuron, 93(1), 15-31. doi: 10.1016/j.neuron.2016.12.003

Hare, T. A., Malmaud, J., \& Rangel, A. (2011). Focusing attention on the health aspects of foods changes value signals in vmpfc and improves dietary choice. Journal of neuroscience, 31 (30), 11077-11087. doi: 10.1523/JNEUROSCI.6383-10.2011

Heeger, D. J. (1992). Normalization of cell responses in cat striate cortex. Visual neuroscience, $9(2), 181-197$. 
Henderson, J. M. (2003). Human gaze control during real-world scene perception. Trends in Cognitive Sciences, 7(11), 498-504. doi: 10.1016/j.tics.2003.09.006

Henderson, J. M., \& Hollingworth, A. (1998). Eye movements during scene viewing: An overview. Eye guidance in reading and scene perception, 269-293.

James, W. (1890/1950). The principles of psychology (Vol. 1). Dover Publications.

Jewell, G., \& McCourt, M. E. (2000). Pseudoneglect: a review and meta-analysis of performance factors in line bisection tasks. Neuropsychologia, 38(1), 93-110.

Kahneman, D. (2003). A perspective on judgment and choice: mapping bounded rationality. American psychologist, 58(9), 697. doi: 10.1037/0003-066X.58.9.697

Kaiser, P. (1991). Flicker as a function of wavelength and heterochromatic ficker photometry. Limits of vision, 171-190.

Kowler, E. (2011). Eye movements: The past 25 years. Vision research, 51(13), 1457-1483.

Krajbich, I., Armel, C., \& Rangel, A. (2010). Visual fixations and the computation and comparison of value in simple choice. Nature Neuroscience, 13(10), 1292-1298. doi: $10.1038 / \mathrm{nn} .2635$

Krajbich, I., \& Rangel, A. (2011). Multialternative drift-diffusion model predicts the relationship between visual fixations and choice in value-based decisions. Proceedings of the National Academy of Sciences, 108(33), 13852-13857. doi: 10.1073/pnas.1101328108

Kruschke, J. (2014). Doing bayesian data analysis: A tutorial with $r$, jags, and stan. Academic Press.

Lee, J., \& Maunsell, J. H. (2009). A normalization model of attentional modulation of single unit responses. PloS one, 4(2), e4651.

Lee, M. D., \& Wagenmakers, E.-J. (2014). Bayesian cognitive modeling: A practical course. Cambridge university press.

Leng, G., Adan, R. A., Belot, M., Brunstrom, J. M., de Graaf, K., Dickson, S. L., . . others (2017). The determinants of food choice. Proceedings of the Nutrition Society, 76(3), 316-327. doi: 10.1017/S002966511600286X

Lim, S.-L., O'Doherty, J. P., \& Rangel, A. (2011). The decision value computations in the vmpfc and striatum use a relative value code that is guided by visual attention. Journal of Neuroscience, 31(37), 13214-13223.

Liu, H.-Z., Zhou, Y.-B., Wei, Z.-H., \& Jiang, C.-M. (2020). The power of last fixation: Biasing simple choices by gaze-contingent manipulation. Acta Psychologica, 208, 103106. doi: $10.1016 /$ j.actpsy.2020.103106

Liu, T., Abrams, J., \& Carrasco, M. (2009). Voluntary attention enhances contrast appearance. Psychological Science, 20(3), 354-362. doi: 10.1111/j.1467-9280.2009.02300.x 
Louie, K., \& Glimcher, P. W. (2012). Efficient coding and the neural representation of value. Annals of the New York Academy of Sciences, 1251(1), 13-32.

Louie, K., Grattan, L. E., \& Glimcher, P. W. (2011). Reward value-based gain control: divisive normalization in parietal cortex. Journal of Neuroscience, 31 (29), 10627-10639.

Luce, R. D., \& Raiffa, H. (1957). Games and decisions [Book]. New York, NY: Dover Publications, Inc.

Mack, A., \& Rock, I. (1998). Inattentional blindness. MIT press.

Macmillan, N. A., \& Creelman, C. D. (2004). Detection theory: A user's guide. Psychology press.

Marois, R., \& Ivanoff, J. (2005). Capacity limits of information processing in the brain. Trends in Cognitive Sciences, 9(6), 296-305.

Masin, S. C., Zudini, V., \& Antonelli, M. (2009). Early alternative derivations of fechner's law. Journal of the History of the Behavioral Sciences, 45(1), 56-65. doi: 10.1002/ jhbs. 20349

McGinty, V. B., Rangel, A., \& Newsome, W. T. (2016). Orbitofrontal cortex value signals depend on fixation location during free viewing. Neuron, 90(6), 1299-1311. doi: 10.1016/ j.neuron.2016.04.045

Mesulam, M.-M. (1981). A cortical network for directed attention and unilateral neglect. Annals of Neurology: Official Journal of the American Neurological Association and the Child Neurology Society, 10(4), 309-325.

Milosavljevic, M., Navalpakkam, V., Koch, C., \& Rangel, A. (2012). Relative visual saliency differences induce sizable bias in consumer choice. Journal of Consumer Psychology, 22(1), 67-74. doi: 10.1016/j.jcps.2011.10.002

Mormann, M., \& Russo, E. J. (2021). Does attention increase the value of choice alternatives. Trends in Cognitive Science, 5(4), 305-315.

Mullett, T. L., \& Stewart, N. (2016). Implications of visual attention phenomena for models of preferential choice. Decision, 3(4), 231. doi: 10.1037/dec0000049

Navalpakkam, V., Koch, C., Rangel, A., \& Perona, P. (2010). Optimal reward harvesting in complex perceptual environments. Proceedings of the National Academy of Sciences, 107(11), 5232-5237. doi: 10.1073/pnas.0911972107

Nittono, H., \& Wada, Y. (2009). Gaze shifts do not affect preference judgments of graphic patterns. Perceptual and Motor Skills, 109(1), 79-94. doi: 10.2466/PMS.109.1.79-94

Orquin, J. L., \& Loose, S. M. (2013). Attention and choice: A review on eye movements in decision making. Acta Psychologica, 144 (1), 190-206. doi: 10.1016/j.actpsy.2013.06.003

Padoa-Schioppa, C. (2009). Range-adapting representation of economic value in the orbitofrontal cortex. Journal of Neuroscience, 29(44), 14004-14014. 
Pärnamets, P., Johansson, P., Hall, L., Balkenius, C., Spivey, M. J., \& Richardson, D. C. (2015). Biasing moral decisions by exploiting the dynamics of eye gaze. Proceedings of the National Academy of Sciences, 112(13), 4170-4175. doi: 10.1073/pnas.1415250112

Pleskac, T. J., Diederich, A., \& Wallsten, T. S. (2015). Models of decision making under risk and uncertainty. In The oxford handbook of computational and mathematical psychology (pp. 209-231). Oxford University Press. doi: 10.1093/oxfordhb/9780199957996.013.10

Pleskac, T. J., Yu, S., Grunevski, S., \& Liu, T. (2022). Attention biases preferential choice by enhancing an option'value. Retrieved 2022-20-28, from https://doi.org/10.17605/ OSF.IO/XQ2KT

Pleskac, T. J., Yu, S., Hopwood, C., \& Liu, T. (2019). Mechanisms of deliberation during preferential choice: Perspectives from computational modeling and individual differences. Decision, 6(1), 77. doi: 10.1037/dec0000092

Plummer, M., et al. (2003). Jags: A program for analysis of bayesian graphical models using gibbs sampling. In Proceedings of the 3rd international workshop on distributed statistical computing (Vol. 124, pp. 1-10).

Posner, M. I. (1980). Orienting of attention. Quarterly Journal of Experimental Psychology, 32(1), 3-25. doi: 10.1080/00335558008248231

Rangel, A., \& Clithero, J. A. (2012). Value normalization in decision making: theory and evidence. Current opinion in neurobiology, 22(6), 970-981.

Ratcliff, R., \& Rouder, J. N. (1998). Modeling response times for two-choice decisions. Psychological Science, 9(5), 347-356. doi: 10.1111/1467-9280.00067

Ratcliff, R., \& Smith, P. L. (2004). A comparison of sequential sampling models for twochoice reaction time. Psychological Review, 111(2), 333-367. doi: 10.1037/0033-295X .111 .2 .333

Ratcliff, R., Smith, P. L., Brown, S. D., \& McKoon, G. (2016). Diffusion decision model: Current issues and history. Trends in cognitive sciences, 20(4), 260-281. doi: 10.1016/ j.tics.2016.01.007

Reeck, C., Wall, D., \& Johnson, E. J. (2017). Search predicts and changes patience in intertemporal choice. Proceedings of the National Academy of Sciences, 114(45), 1189011895. doi: 10.1073/pnas.1707040114

Reynolds, J. H., \& Heeger, D. J. (2009). The normalization model of attention. Neuron, $61(2), 168-185$.

Rinaldi, L., Di Luca, S., Henik, A., \& Girelli, L. (2014). Reading direction shifts visuospatial attention: An interactive account of attentional biases. Acta psychologica, 151, 98-105.

Shakespeare, W. (1853). Romeo and juliet. Enslin.

Shimojo, S., Simion, C., Shimojo, E., \& Scheier, C. (2003). Gaze bias both reflects and influences preference. Nature Neuroscience, 6(12), 1317-1322. doi: 10.1038/nn1150 
Simion, C., \& Shimojo, S. (2006). Early interactions between orienting, visual sampling and decision making in facial preference. Vision research, $46(20), 3331-3335$. doi: 10.1016/ j.visres.2006.04.019

Simion, C., \& Shimojo, S. (2007). Interrupting the cascade: Orienting contributes to decision making even in the absence of visual stimulation. Perception \& psychophysics, $69(4), 591-595$. doi: 10.3758/BF03193916

Simons, D. J., \& Chabris, C. F. (1999). Gorillas in our midst: Sustained inattentional blindness for dynamic events. perception, 28(9), 1059-1074.

Smith, S. M., \& Krajbich, I. (2019). Gaze amplifies value in decision making. Psychological Science, 30(1), 116-128. doi: 10.1177/0956797618810521

Spiegelhalter, D. J., Best, N. G., Carlin, B. P., \& Van der Linde, A. (2014). The deviance information criterion: 12 years on. Journal of the Royal Statistical Society: Series B: Statistical Methodology, 485-493.

Steyvers, M. (2011). Matjags 1.3: A matlab interface for jags.

Summerfield, C., \& Tsetsos, K. (2012). Building bridges between perceptual and economic decision-making: Neural and computational mechanisms. Frontiers in Neuroscience, 6 , 70. doi: $10.3389 /$ fnins.2012.00070

Tavares, G., Perona, P., \& Rangel, A. (2017). The attentional drift diffusion model of simple perceptual decision-making. Frontiers in Neuroscience, 11, 468. doi: 10.3389/ fnins. 2017.00468

Theeuwes, J. (2004). Top-down search strategies cannot override attentional capture. Psychonomic bulletin \& review, 11(1), 65-70.

Theeuwes, J., Kramer, A. F., Hahn, S., Irwin, D. E., \& Zelinsky, G. J. (1999). Influence of attentional capture on oculomotor control. Journal of experimental psychology: human perception and performance, 25(6), 1595.

Thomas, A. W., Molter, F., Krajbich, I., Heekeren, H. R., \& Mohr, P. N. (2019). Gaze bias differences capture individual choice behaviour. Nature human behaviour, 3(6), 625-635. doi: $10.1038 /$ s41562-019-0584-8

Thut, G., Nietzel, A., Brandt, S. A., \& Pascual-Leone, A. (2006). $\alpha$-band electroencephalographic activity over occipital cortex indexes visuospatial attention bias and predicts visual target detection. Journal of Neuroscience, 26(37), 9494-9502.

Towal, R. B., Mormann, M., \& Koch, C. (2013). Simultaneous modeling of visual saliency and value computation improves predictions of economic choice. Proceedings of the National Academy of Sciences, 110(40), E3858-E3867. doi: 10.1073/pnas.1304429110

Tsetsos, K., Chater, N., \& Usher, M. (2012). Salience driven value integration explains decision biases and preference reversal. Proceedings of the National Academy of Sciences, 109(24), 9659-9664. doi: 10.1073/pnas.1119569109 
Turatto, M., Vescovi, M., \& Valsecchi, M. (2007). Attention makes moving objects be perceived to move faster. Vision Research, $47(2), 166-178$. doi: 10.1016/j.visres.2006.10 .002

Von Helmholtz, H. (1925). Helmholtz's treatise on physiological optics (Vol. 3). Optical Society of America.

Wabersich, D., \& Vandekerckhove, J. (2014). Extending jags: A tutorial on adding custom distributions to jags (with a diffusion model example). Behavior Research Methods, 46(1), $15-28$.

Weber, E. U., \& Johnson, E. J. (2009). Mindful judgment and decision making. Annual Review of Psychology, 60, 53-85. doi: 10.1146/Annurev.Psych.60.110707.163633

Wichmann, F. A., \& Hill, N. J. (2001). The psychometric function: I. fitting, sampling, and goodness of fit. Perception 83 psychophysics, 63(8), 1293-1313. doi: 10.3758/ BF03194544

Yantis, S. (1993). Stimulus-driven attentional capture. Current Directions in Psychological Science, 2(5), 156-161.

Zajonc, R. B. (1968). Attitudinal effects of mere exposure. Journal of Personality and Social psychology, 9(2p2), 1-27. doi: 10.1037/h0025848

Zajonc, R. B. (2001). Mere exposure: A gateway to the subliminal. Current Directions in Psychological Science, 10(6), 224-228. doi: 10.1111/1467-8721.00154

Zeigenfuse, M. D., Pleskac, T. J., \& Liu, T. (2014). Rapid decisions from experience. Cognition, 131(2), 181-194. doi: 10.1016/j.cognition.2013.12.012

Zoltak, M. J., Veling, H., Chen, Z., \& Holland, R. W. (2018). Attention! can choices for low value food over high value food be trained? Appetite, 124, 124-132. doi: 10.1016/ j.appet.2017.06.010 